\title{
Transmission Dynamics and Underreporting of Kala-azar in the Indian State of Bihar
}

\author{
Anuj Mubayi ${ }^{\mathrm{a}, \mathrm{b}, \mathrm{d}, \mathrm{f}, *, 1}$, Carlos Castillo-Chavez ${ }^{\mathrm{a}, \mathrm{b}, \mathrm{c}, \mathrm{e}}$, Gerardo Chowell ${ }^{\mathrm{a}, \mathrm{b}}$, Christopher \\ Kribs-Zaleta $^{\mathrm{d}}$, Niyamat Ali Siddiqui ${ }^{\mathrm{g}}$, Narendra Kumar ${ }^{\mathrm{g}}$, Pradeep Das ${ }^{\mathrm{g}}$ \\ ${ }^{a}$ Mathematical $\&$ Computational Modeling Sciences Center, Arizona State University, AZ, USA \\ ${ }^{b}$ School of Human Evolution and Social Change, Arizona State University, AZ, USA \\ ${ }^{c}$ School of Mathematical and Statistical Sciences, Arizona State University, AZ, USA \\ ${ }^{d}$ Department of Mathematics, The University of Texas at Arlington, TX, USA \\ ${ }^{e}$ Santa Fe Institute, Santa Fe, NM, USA \\ ${ }^{f}$ Prevention Research Center, Berkeley, CA, USA \\ ${ }^{g}$ Rajendra Memorial Research Institute of Medical Sciences (ICMR), Patna, Bihar, India
}

\begin{abstract}
"Kala-azar" (or Indian Visceral Leishmaniasis) is a vector-borne infectious disease affecting communities in tropical and subtropical areas of the world. Bihar, a state in India, has one of the highest prevalence and mortality reported levels of Kala-azar. Yet, the magnitude of the problem is difficult to assess because most cases are handled by private health providers who are not required to and do not report them to the Ministry of Health. The impact of underreporting using district-level reported incidence data from the state of Bihar is the main goal of this manuscript. We derive expressions for, and compute estimates of Kala-azar's reproduction num-

\footnotetext{
${ }^{*}$ Corresponding author

Email addresses: mubayi@uta.edu (Anuj Mubayi), chavez@math.la.asu.edu (Carlos Kribs-Zaleta), niyamatalisiddiqui@yahoo.com (Niyamat Ali Siddiqui), narendra54in@yahoo.com (Narendra Kumar), drpradeep.das@gmail.com (Pradeep Das)

${ }^{1}$ Department of Mathematics, The University of Texas at Arlington, Arlington, TX 76019-0408; Phone: 480-209-5329; Fax: 817-272-5802
} Castillo-Chavez ), gchowell@asu.edu (Gerardo Chowell), kribs@uta.edu (Christopher
\end{abstract}


bers, an indirect measure of disease prevalence, and levels of underreporting for the 21 districts of Bihar. The average reproduction number (number of secondary cases generated per infective) estimates for Bihar range from 1.3 (2003) to 2.1 (2005) with some districts' estimates with mean values lower than one. Model estimates (using available data and a model-derived expression) show that the proportion of underreported cases declined from an average of $88 \%$ in 2003 to $73 \%$ in 2005 . However, 8 districts in 2003 and 5 districts in 2005 had more than $90 \%$ levels of underreporting. Model estimates are used to generate underreporting adjusted incidence rates. The analysis finds that reported data misidentify four of the eight (2003) and three of the nine (2005) districts classified as high-risk. In fact, seven (2003) and five (2005) of the most affected Kala-azar districts had been classified as low-risk when only reported incidence data were used.

Key words: Leishmaniasis, Mathematical Model, Reproduction Number, Private Health Care Providers; Public Health Surveillance Systems.

\section{Introduction}

Leishmaniasis is a family of infectious diseases caused by parasites of the Leishmania genus that spreads in mammals (human and animal hosts) through the bites of sandflies. The geographical distribution of Leishmaniasis is tied in to the abundance of sandflies, their life cycle, and the presence of the parasite reservoirs. The Leishmaniasis is classified from its clinical manifestation (Visceral Leishmaniasis (VL), Cutaneous Leishmaniasis (CL), Muco-Cutaneous Leishmaniasis (MCL) or Diffuse-Cutaneous Leishmaniasis (DCL)) and host-reservoir (Anthroponotic (A) or Anthropo-zoonotic (AZ) or Zoonotic (Z)) [30]. The world's Leishmaniasis prevalence 
is between 10-12 million while the incidence of clinical cases is between 1.5 and 2.5 million (including 500,000 new cases of VL) each year [18, 27, 37, 38, 52, 55]. The World Health Organization (WHO) classifies Leishmaniasis as a neglected disease affecting the poorest communities around the world. It is believed that about $30 \%$ of the new clinical cases ([55]) and around 58,000 Leishmaniases-indiced deaths (mostly attributed to VL; $[53,54,56])$ are reported worldwide each year. Ninety per cent of worldwide VL cases, the most severe form of the disease, occur in Bangladesh, India (mainly, northeastern region), Nepal, Sudan and Brazil (northeastern region). VL may infect 300,000 people annually causing over 20,000 deaths, each year in India alone $[44,46]$.

Locally known as "Kala-azar" (Hindi for 'black fever'), VL, in India is considered to be anthroponotic, and it is nearly always fatal if untreated within 2 to 3 years after infection. The likelihood of deaths among patients receiving treatment is between $4 \%$ and $15 \%$ [7] with the rest recovering with immunity to re-infection albeit, occasional relapses have been documented [25]. Drugs used to treat Kala-azar, developed more than 60 years ago, include pentavalent antimonial compounds $\left(S b^{v}\right)$. These drugs are no longer effective in Bihar due to high levels of resistance in the population. Alternative therapeutic measures are available but with varying level of effectiveness and side effects (see Table S.4). Bihar is believed to house more than $90 \%$ of India's Kala-azar burden [7, 37] (or about 270,000 new cases per year). On the other hand, the official number of reported cases (deaths) in Bihar was approximately 14,000 (200) in 2003 and 23,000 (125) in 2005. Reporting has been estimated (guessed) to be between $5 \%$ and $8 \%$ of the total number of estimated cases in Bihar. 
A mathematical model of Anthroponotic Visceral Leishmaniasis (AVL) or Kalaazar transmission at the district-level is introduced. Estimates of the local reproduction number are generated from the model as well as an estimate $\hat{p}$ of the proportion of reported cases. Reported incidence data (2003 and 2005) are used to obtain a distribution of model-dependent underreporting levels per district. We compare reported incidence with computed model-adjusted incidence rates. Correlations between these incidence (reported and adjusted) estimates and district demographic and socioeconomic factors (Table 1) are calculated.

- - - - - Insert Table 1 about here. - - - -

There are plenty of studies of the dynamics of vector-borne diseases using mathematical models (e.g., Dengue [22]; Malaria [28]; West Nile virus [49]). The only mathematical study of the dynamics of AVL (Kala-azar) appears to be that of Dye and Wolpert in 1988 [21]. They used a deterministic dynamical model to explain the observed AVL inter-epidemic periods between 1875 and 1950 in Assam, India. Their study concluded that recurrent outbreaks (every 10 to 15 years) appear to be the result of intrinsic factors (vector and host dynamics, birth and death rates, population immunity) and not extrinsic processes (drugs, natural disasters, other infectious diseases). Qualitative studies of the dynamics of Leishmaniasis have focused on either ZCL (zoonotic CL) or ZVL (zoonotic VL) e.g. [2, 8, 12, 15, 16, 19, 20, 32]. 


\subsection{Data Sources}

Government agencies in India collect data via passive case detection ${ }^{2}[18]$ which grossly underestimates the number of cases. Most cases are diagnosed but not reported by private medical practitioners. Private doctors are not required to report cases to the state health system. The social and cultural stigma linked to VL and high prevalence among the poor results in a large percentage of individuals failing to seek treatment at public health facilities [33]. Hence, it is not possible to obtain directly reliable estimates of VL incidence using the surveillance system in place. The focus of this paper is to quantify the reproduction numbers and the levels of underreporting via modeling framework and, if possible, to identify improvements in the surveillance system.

The state system (State Health Society) of Bihar is divided into 38 District Health Societies (DHS). Each DHS is further subdivided into a variable number of blockPrimary Health Centers (PHCs). PHC consists of Sub-Centers providing health care to a variable number of villages (e.g. the Muzaffarpur district (population 3.7 million) has 14 Block PHCs while the Kanti Block (population 337,670 [10]) has 48 subcentres, each covering a population of roughly 8000 individuals [38]). PHCs, district hospitals and government medical colleges are the sources of reported cases [31]. The private health sector includes not-for-profit and for-profit organizations. Forprofit venues include corporations (e.g., private nursing homes), trusts, stand-alone specialist services, pharmacy shops, and self-appointed practitioners. Estimates sug-

\footnotetext{
${ }^{2}$ Individuals who seek treatment from the public health system are the only cases officially reported. Active case detection policies require that new cases are recorded by public health officials. These officials frequently visit local regions and inquire about new cases.
} 
gest that $80 \%$ of the outpatients and $57 \%$ of the inpatients are handled in the private sector (2001 World Bank report, [50]). NGOs usually provide awareness and education programs, carry out research, and provide access to regular health services. Ninety per cent of Bihar's population lives in rural areas where less than $1 \%$ of health services are provided by not-for-profit/Non-Government Organizations (NGOs) [50].

The monthly number of reported Kala-azar cases from Bihar's 21 most affected districts (out of 38 districts) as well as from the state during 2003 and 2005 are used in this study. Data sets belong to the State Health Society of Bihar and access was provided by the Rajendra Memorial Institute of Medical Sciences. Leishmaniasis occurs in annual epidemics because of seasonal fluctuations in sandfly populations [2, 21, 48]. Annual data is used to compute 2003 and 2005 epidemic estimates (data for 2004 was not available). It is worth observing that 2003 and 2005 data were collected under a different set of public health policies: 2003 under the status $q u o^{3}$ and 2005 under improved health policies. This improved public health program is because of the formation of Kala-azar Task Force that was put in place by the Government of India and the WHO, in December of 2003. Under this program tools were made available for simple, and inexpensive tests for field-level diagnosis, a new oral drug (Miltefosine) that is easy to administer at community level, free diet and incentive to patient and one of his attendant, and encouragement to kalaazar activists for case detection [31]. We use data from 2003 (regular public health program) and 2005 (improved public health program) to evaluate the effects of the program.

\footnotetext{
${ }^{3}$ regular control policies
} 
The difference in the district-wise monthly reported incidence rates from 2003 to 2005 are highlighted in Figure S.4. The reported incidence rates of roughly half of the 21 districts are larger than the average Bihar rates of 16.2 in 2003 and 26.0 in 2005. Monthly reported incidence rates in 2005 are higher than 2003 rates for all but 5 of the 21 districts (Figures S.3). The reported-incidence peak rates took place between April and September (Figures S.3), around the monsoon period, when the density of sandflies increases.

Data on population density, population size in rural and urban areas, literacy rates, and number of public health medical facilities in each of the 21 districts are generated from the 2001 census [10], under the assumptions that the population of each district increases at an annual rate of $2.1 \%$ (see [10]).

Section 2 describes the model, model assumptions, and the parameter estimation techniques used. Section 3 includes the basic mathematical analysis of the model and a method for quantifying underreporting. Estimates of the reproduction numbers and underreporting levels are found in Section 4. Section 5 includes a discussion of the results and some of their implications.

\section{Model Description}

A system of nonlinear coupled differential equations is used to model the transmission dynamics of Kala-azar that incorporates the appropriate epidemiological states, and transmission mechanisms. The model framework allows for the incorporation of long latent and infectious periods, and two modes of transmissions. Furthermore, the model incorporates the possibility that individuals seek treatment at private or 
public health facilities. The infectious period is variable (20 days to 1 year [29]); the incubation period is between 2 and 6 months [51]; and the average treatment periods is also variable in Bihar (Table S.4). These variations in epidemiological factors are easily captured via a "Staged-Progression Models" (SPM) [14, 23].

\subsection{Model Structure}

The model (Figure 1) assumes that individuals are born susceptible $(S)$ at the rate $\mu N_{h}$, where $N_{h}$ is the size of the human population. Individuals' lifespan is assumed to be exponentially distributed with an average (life expectancy) of $1 / \mu$ years. Once a susceptible individual gets infected through the bite of an infected sandfly, he/she becomes latent and progresses through $n_{1}$ latent stages $\left(E_{i}, i=1, \ldots, n_{1}\right)$ before becoming infectious. Latent individuals (by assumption) spend exponentially distributed times in each latent stage, that is, the latent period (assumed same as the incubation period in the model $)$ distribution is given by the $\Gamma\left(n_{1}, n_{1} \gamma_{h}\right)$ distribution with mean $1 / \gamma_{h}$ and standard deviation of $1 /\left(\gamma_{h} \sqrt{n_{1}}\right)$ (Supplementary Document). Progression to full-blown Kala-azar leads to an infectious state $\left(I_{i}\right)$. The infectious period distribution is modeled by the $\Gamma\left(m_{1}, m_{1} \eta_{h}\right)$ distribution with mean $1 / \eta_{h}$ and standard deviation $1 /\left(\eta_{h} \sqrt{m_{1}}\right)$. Infectious individuals are assumed to be equally infectious through all $m_{1}$ stages $\left(I_{i}, i=1, \ldots, m_{1}\right)$.

\section{- - - - - Insert Figure 1 about here. - - - - -}

Infectious individuals, if untreated, within 2 to 3 years following infection are unlikely to survive [7] while death rates among patients receiving complete treatment are extremely low. Individuals undergoing treatment in government or private health 
facilities are grouped in the classes $G_{i}, i=1, \ldots, k_{1}$ and $T_{j}, j=1, \ldots ., k_{2}$, respectively. It is assumed that all individuals undergo treatment. Hence, the parameter " $p$ " denotes the fraction of patients undergoing treatment in public health facilities and $(1-p)$ the treated fraction at private health venues. Individuals treated at public or private health facilities recover at the per-capita rates $\alpha_{h}$ and $\widetilde{\alpha}_{h}$, respectively, moving to the recovered class $R$, where they acquire "life-long" immunity. We model $\widetilde{\alpha}_{h}$ via $\widetilde{\alpha}_{h} \equiv q \times \alpha_{h}$ where $q \in[0,1] ; q=1$ means that the length of treatment period in public and private treatment facilities are the same but $q=0.75$ means that the average treatment length in private facilities is $4 / 3$ (i.e., $1 / q$ ) times longer.

During the infectious period an individual may be bitten by susceptible female sandflies (class $X)$. Infected vectors move through $n_{2}$ latent stages $\left(Y_{i}, i=1, \ldots, n_{2}\right)$ before becoming infectious (class $Z$ ). The extrinsic latent period is $1 / \gamma_{v}$. Total birth and death rates of the vector population are assumed to be equal, that is, the size of the vector population, $N_{v}$, is constant. The average life-span of a vector is $1 / \mu_{v}$ units of time. $E(t)=\sum_{i_{1}=1}^{n_{1}} E_{i_{1}}(t), I(t)=\sum_{j_{1}=1}^{m_{1}} I_{j_{1}}(t), G(t)=\sum_{l_{1}=1}^{k_{1}} G_{l_{1}}(t)$, $T(t)=\sum_{l_{2}=1}^{k_{2}} T_{l_{2}}(t)$ and $Y(t)=\sum_{i_{2}=1}^{n_{2}} Y_{i_{2}}(t)$ denote the total numbers of latent individuals, infectious individuals, Kala-azar patients being treated at government health clinics, Kala-azar cases getting treatment at private facilities, and infected vectors in the latent stage, respectively.

The rate of infection for susceptible hosts is modeled by $F_{h}(t)=\lambda_{h} \frac{Z(t)}{N_{v}(t)}$ with $\lambda_{h} \equiv$ $m C \beta_{h v}$. Here, $m$ is the per-capita average number of sandflies (assumed constant), $C$ is the mean rate of bites per sandfly, $\beta_{h v}$ is the transmission "probability" per bite from an infectious sandfly, and $\frac{Z}{N_{v}}$ is the proportion of infectious sandflies in the 
vector population. AVL is a deadly disease among the untreated and the population of Bihar is large and changing (because of migration [17]). However, the data cover only two years, for 2003 and 2005. Hence, taking $N_{h}$ to be constant is roughly acceptable. Furthermore, typically, adult vector populations sizes or densities are limited by the mortality rates experienced in the early stages of the life history of the sandfly. Hence, in areas where there are no drastic changes in adult sandfly population sizes or densities, the assumption of constant $N_{v}$ is probably acceptable.

The proportion of bites of susceptible sandflies on infectious humans is modeled by $\frac{\sum_{j_{1}=1}^{m_{1}} I_{j_{1}}}{N_{h}}$, that is, sandflies bite the host population at random. The infection rate of susceptible sandflies is $F_{v}(t)=\lambda_{v} \frac{\sum_{j_{1}=1}^{m_{1}} I_{j_{1}}(t)}{N_{h}(t)}$ with $\lambda_{v}=C \beta_{v h}$ where $\beta_{v h}$ is the transmission "probability" per infectious human bite by a susceptible sandfly. $\frac{\sum_{j_{1}=1}^{m_{1}} I_{j_{1}}(t)}{N_{h}(t)}$ is the "probability" that a sandfly bites a randomly selected infectious human [14].

\subsection{Model Parameters}

The period of parasite sequential development in a sandfly lasts from 3 to 7 days $[35,36]$ and so, it is assumed that vector latent period is roughly between 3 and 7 days. The average life of an adult sandfly in Bihar is 14 days (95\% CI: $(1.5,26.5))$ [39]. The average treatment period in public health facilities, $1 / \alpha_{h}$, is calculated using a weighted average with weights tied in to efficacy estimates (given in Table S.4) of available therapeutic measures. There is a large proportion of unqualified medical practitioners working in non-governmental health facilities who may over or under prescribe (usually over prescribe) drugs. It is assumed that the average treatment length in private health facilities is $4 / 3$ times the one in public facilities 
$(q=0.75)$ but this parameter seems not important as the model results are not sensitive to variations in $q$ in the "expected range".

\section{- - - - Insert Table 2 about here. - - - - -}

Estimates and ranges of some model epidemiological parameters are taken from the literature (Table 2). The latent period range for humans is between 2 and 6 months (additional estimates, all in same range, are found in [3], [7], and [51]). An average infectious period of 3.80 months (standard deviation of 3.55 months) are taken from [40]; its 95\% confidence intervals (assuming normally distributed infectious period) includes other estimates of infectious period (4.47 months, Ethopia [1]; 4.88 months, India [11]; 3.18 months, Bihar [38] and the range 5.3-6.7 months, Chandigarh [29]). The average life span of the adult sandfly is about 14 days (standard deviation 12.5 days) [39]. These estimates are comparable to others in the literature (8.6 days with $95 \%$ CI $(4,15)$ days) in $[24])$.

The average number of class-stages (i.e., $n_{1}, m_{1}, n_{2}, k_{1}$ and $k_{2}$ ) is estimated from the standard deviation and the average estimate of the corresponding epidemiological parameters (Table 2). For example, the number of infectious stages, $m_{1}$, is computed from the statistics of the infectious period as follows. Since the infectious period follows the gamma distribution, $\Gamma\left(m_{1}, m_{1} \eta_{h}\right)$, with mean $\left(1 / \eta_{h}\right)$ of 3.80 months and the standard deviation (std) of 3.55 months therefore

$$
\begin{aligned}
\operatorname{std}(\text { Infectious period }) & =\frac{1}{\eta_{h} \sqrt{m_{1}}}=3.55 \\
\Longrightarrow m_{1} & =\left(\frac{3.80}{3.55}\right)^{2} \approx 2 .
\end{aligned}
$$


The values of $k_{1}, k_{2}, n_{1}$ and $n_{2}$ are estimated in the same way.

Monthly reported case data from 2003 and 2005 are used to generate initial estimates of $\lambda_{h}, \lambda_{v}, E_{1}(0)$, and $I_{1}(0)$, for each district, and each year (2003 and 2005). The "best" fit of the model (with $p$ at 0.12 [38]) to the cumulative number of reported Kala-azar cases per district (official data) using a least-square fitting procedure in MATLAB (built-in routine lsqcurvefit found in the optimization toolbox) is used to generate these four estimates. Not only $p$ but also other model parameters (given in Table 2) are kept fixed (mean values) during the estimation procedure.

The above estimation procedure is repeated 8 times (using 5, 6, 7, 8, 9, 10, 11 or 12 data points, the year starts in January) for each district in 2003 and 2005. We use the Chi-square goodness-of-fit statistical test to select the best fitting parameters. The parameter-set (among the 8 sets of parameter estimates) that gives a minimal $\chi^{2}$-density is selected for each district (see details in [14]). The $\chi^{2}$-statistic was calculated from the formula

$$
\chi^{2}=\sum_{i=1}^{n}\left\{\frac{\left(x_{i}-g_{i}\right)^{2}}{g_{i}}\right\}
$$

where $i=1$ is the month of January, ' $n$ ' is the number of data points (each corresponding to 12 months) used in the estimation, $x_{i}$ s are data points (cumulative), and $g_{i} \mathrm{~S}$ are points on the solution curve (cumulative case treated at government health facilities) of the model fitted to the cumulative data. The test statistic follows, approximately, a chi-square distribution with $(n-4)$ degrees of freedom because in each case we estimated 4 parameters. 


\section{Key Quantities Derived from the Model}

We re-scale the system by setting $s \equiv \frac{S_{h}}{N_{h}}, x \equiv \frac{X}{N_{v}}$, etc. The re-scaled system supports two steady states: the Disease-Free State (DFS) and the Endemic State (ES) (Supplementary Document).

\subsection{Reproduction Number and Model Analysis}

Zoonotic Leishmaniasis mathematical studies have computed the "basic"- $\left(R_{0}\right)$, "control"- $\left(R_{*}\right)$, and "effective"- $\left(R_{e}\right)$ reproduction numbers $[2,8,12,16]$. The reproduction number quantifies whether a disease will persist in the population or not and typically if its value is less than one, the disease dies out but if it is greater than one the disease becomes endemic. $R_{0}$ is computed in the absence of control measures when no one in the population is immune to the disease. The effective reproduction number $\left(R_{e}\right)$ gives the average number of secondary cases generated by

a disease in a population, at a particular time $t$. Roughly, $R_{e}(t) \cong R_{0} \times \frac{S(t)}{N_{h}}$. The control reproduction number $\left(R_{*}\right)$, the average number of secondary cases generated by primary cases under specified controls, roughly estimated by $R_{*} \cong R_{0}(1-h)$, where $h$ captures the reduction in $R_{0}$ from the interventions measures. AVL is endemic in the regions of interest, hence, we define $R_{c}$, the reproduction number, by $R_{c} \cong(1-\nu) R_{*}$ where $\nu$ is the proportion of the population effectively protected (herd immunity) at the start of the outbreak. $R_{c}$ computed ([47]) from the model as

$$
\begin{aligned}
R_{c} & \equiv \sqrt{\left(\frac{n_{1} \gamma_{h}}{n_{1} \gamma_{h}+\mu_{h}}\right)^{n_{1}}\left[\sum_{i=1}^{m_{1}} \frac{\lambda_{v}}{\left(m_{1} \eta_{h}+\mu_{h}\right)}\left(\frac{m_{1} \eta_{h}}{m_{1} \eta_{h}+\mu_{h}}\right)^{i-1}\right]\left(\frac{n_{2} \gamma_{v}}{n_{2} \gamma_{v}+\mu_{v}}\right)^{n_{2}}\left(\frac{\lambda_{h}}{\mu_{v}}\right)} \\
& =\sqrt{\left(\frac{B_{1}}{1-A_{1}}\right)\left(\frac{A_{1}}{1-B_{1}}\right)} .
\end{aligned}
$$


where $A_{1}=\frac{\lambda_{v} A B}{\lambda_{v} A B+\mu_{v}}, B_{1}=\frac{\lambda_{h} P_{3}^{n_{2}}}{\lambda_{h} P_{3}^{n_{2}}+\mu_{h}}, A=\left(P_{2}\right)^{n_{1}} \times\left(1-P_{1}\right), B=\sum_{i=0}^{m_{1}-1}\left(P_{1}\right)^{i}$, $P_{1}=\left(\frac{m_{1} \eta_{h}}{m_{1} \eta_{h}+\mu_{h}}\right), P_{2}=\left(\frac{n_{1} \gamma_{h}}{n_{1} \gamma_{h}+\mu_{h}}\right)$, and $P_{3}=\left(\frac{n_{2} \gamma_{v}}{n_{2} \gamma_{v}+\mu_{v}}\right)$. The components of $R_{c}$ are:

- $\left(\frac{n_{1} \gamma_{h}}{n_{1} \gamma_{h}+\mu_{h}}\right)^{n_{1}}$ is the proportion of infected humans that survive the latent period.

- $\sum_{i=1}^{m_{1}} \frac{\lambda_{v}}{\left(m_{1} \eta_{h}+\mu_{h}\right)}\left(\frac{m_{1} \eta_{h}}{m_{1} \eta_{h}+\mu_{h}}\right)^{i-1}$ represents the average number of infected vectors generated by infectious humans over their entire infectious period.

- $\left(\frac{n_{2} \gamma_{v}}{n_{2} \gamma_{v}+\mu_{v}}\right)^{n_{2}}$ is the proportion of infected vectors surviving their latent period.

- $\left(\frac{\lambda_{h}}{\mu_{v}}\right)$ represents the number of newly infected humans generated per infectious vector.

The DFS is always a viable steady state, and it is locally asymptotically stable if $R_{c}<1$. When $R_{c}>1$ two states (DFS and ES) coexist, DFS unstable. Numerical simulations suggest that ES is not only locally asymptotically stable but that the disease becomes endemic whenever $R_{c}>1$.

$R_{c}$, given by Equation (1), does not depend on the reporting proportion $(p)$ or on the treatment rates $\left(\alpha_{h}\right.$ and $\left.\widetilde{\alpha}_{h}\right) . R_{c}$ depends on the average length of the effective infectious period (modified by treatments), the contact rate, the intrinsic and extrinsic average latent periods and the susceptible proportion. Increases in the per-capita treatment rates reduce the average length of the effective infectiousness decreasing the value of the effective reproduction number. The impact of vector control measures (e.g., spraying of insecticides) are typically evaluated from their impact on $R_{c}$ (relative to $R_{0}$ ). 


\subsection{Computation of Underreporting Percentage}

Kala-azar is endemic in Bihar and therefore, the rate at which new cases seek treatment at public health facilities (i.e., rate of reporting of cases) is $p m_{1} \eta_{h} I_{m_{1}}^{*}$ ( $I_{m_{1}}^{*}$ the endemic state value of the last infectious class). If the units of this rate are individuals per month then it must be equal to the average number of reported cases per month. Equating this rate with the actual rate of reported cases per month leads to an expression for $p$ in terms of $m_{1}, \eta_{h}, I_{m_{1}}^{*}$, and the monthly reported cases. We find that

$$
p=\frac{(\text { Reported Cases per Month) }}{\left(\frac{m_{1} \eta_{h}}{k_{1} \alpha_{h}+\mu_{h}}\right)\left(P_{1}\right)^{m_{1}-1}\left(P_{2}\right)^{n_{1}}\left(1-P_{1}\right)\left(1-\frac{1-B_{1}}{A_{1}}\right)}
$$

where $A_{1}=\frac{\lambda_{v} A B}{\lambda_{v} A B+\mu_{v}}, B_{1}=\frac{\lambda_{h} P_{3}^{n_{2}}}{\lambda_{h} P_{3}^{n_{2}}+\mu_{h}}, A=\left(P_{2}\right)^{n_{1}} \times\left(1-P_{1}\right), B=\sum_{i=0}^{m_{1}-1}\left(P_{1}\right)^{i}$, $P_{1}=\left(\frac{m_{1} \eta_{h}}{m_{1} \eta_{h}+\mu_{h}}\right), P_{2}=\left(\frac{n_{1} \gamma_{h}}{n_{1} \gamma_{h}+\mu_{h}}\right)$, and $P_{3}=\left(\frac{n_{2} \gamma_{v}}{n_{2} \gamma_{v}+\mu_{v}}\right)$.

$p$ depends on $\lambda_{h}$ (in $B_{1}$ ) and $\lambda_{v}$ (in $A_{1}$ ), which have been estimated for the 21 districts of interest. Using model estimates and data we compute an "empirical" distribution for $p$ for each district as follows:

The parameters $\eta_{h}, \mu_{h}, \gamma_{h}, \gamma_{v}$ and $N_{h}$ are fixed (Table 2). Distributions are assigned to the parameters $\mu_{v}, \lambda_{v}, \lambda_{h}, m_{1}, n_{1}$ and $n_{2}$ based on "experience." The parameter $1 / \mu_{v}$ (average life span of the vector) follows an inverse gamma distribution with the mean and standard deviation in Table 2; and, the parameters $\lambda_{h}$ and $\lambda_{v}$ are assigned exponential distributions with means as earlier estimates. The variability in the infectious period, intrinsic and extrinsic incubation/latent periods, is in the distributions for $m_{1}, n_{1}$ and $n_{2}$, respectively. Discrete (integer value) uniform 
distributions are chosen for $m_{1}, n_{1}$ and $n_{2}$ with estimated ranges in the intervals $[1,3],[4,28]$ and $[11,39]$, respectively (see references in Table 2 and references $[1,3,4,7,29,36])$.

For each district, the variable Reported Cases is assumed to be uniformly distributed. The lower and upper limits of this uniform distribution are computed from reported incidence in such a way as to make the interval both symmetric about the mean, and a subset of the interval into which the real data fall. The variation from the mean to the upper and lower limits of the distribution is taken to be the smaller of the distances from the mean to the minimum and maximum values assumed by the reported case data for that district. A conditional sample (conditioned on $R_{c}>1$ ) is chosen from these distributions using Monte-Carlo sampling $[6,13]$. The sample set is plugged in Equation (3) to generate a single estimate of $p$. This process is repeated $10^{4}$ times choosing independently a sample of parameters (paired at random) each time. The average estimate of $p$ (i.e., $\operatorname{Mean}(p)=\hat{p}$ ) is computed from these $10^{4}$ samples but more generally, we generate an empirical (model-dependent) distribution for $p$.

\section{Results}

\subsection{Estimates of The Control Reproduction Number}

Each of the 21 districts and Bihar's best parameter estimates for $\lambda_{h}, \lambda_{v}, E_{1}(0)$ and $I_{1}(0)$ are obtained for 2003 and 2005 (Table S.6). These estimates (using reported incidence data) are used to compute estimates of the reproduction number $\left(R_{c}\right)$ for all

21 districts (Table S.8). $R_{c^{-}}$-estimates for Bihar are obtained in two ways: averaging 
district-estimates over all 21 districts $\left(R_{c}^{*}\right)$ or from aggregated reported state data $\left(R_{c}\right)$. These estimates are in Table S.6. We observed $R_{c}$-estimates less than 1 in 2003 for some districts where the infection was found to be endemic in 2005. The use of local $R_{c}$ values implicitly assumes that the districts are "disconnected" but in fact, there is high movement of workers between neighboring districts [16, 17], so local values less than one are not unusual. $R_{c}$-estimates greater than one are found for 11 districts (2003) and 4 districts (2005) where Kala-azar is endemic (Patna and Saharsa are in both lists). $R_{c^{-}}$estimates are highest in Samastipur (2003) and Saharsa

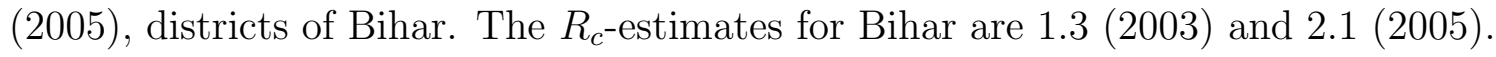

\subsection{Underreporting in Districts}

Districts are ranked according to their estimated average levels of underreporting in 2003 and 2005 (Table 3). Most of these averages decreased except for the districts of Jahanabad, Madhubani, Samastipur, and Katihar. The biggest decrease took place in Vaishali (74.9\% in 2003 and $44.7 \%$ in 2005) and the largest increase in Katihar (65.1\% in 2003 and $78.4 \%$ in 2005). The lowest number of reported cases took place in Jahanabad (4\% in both 2003 and 2005) and the highest in Madhepura (56\% in 2003 and $32 \%$ in 2005). High underreporting (>90\%) was found in 8 districts in 2003 and 5 in 2005. Patna, Nalanda, and Jahanabad belonged to the class of high

underreporting in both years (Table 3). Bihar's overall underreporting declined by about $17 \%$ from $88 \%$ in 2003 to $73 \%$ in 2005 .

- - - - Insert Table 3 about here. - - - - 
Model results and model-generated estimates are used to explore potential correlations between underreporting and demographic and socioeconomic variables such as population size, population density, urbanization levels (fraction of the district population living in rural areas), literacy rates, and number of public health facilities per million residents. No significant correlation was found between the model-generated underreporting estimates and population size-dependent measures (rural community size, number of PHC, Sub-Centers, or Medical Facilities per million residents) at the district level $(n=21)$ in 2003 and 2005. However, population density (i.e., population per square $\mathrm{km}$ ) is positively correlated with model-generated underreporting estimates (see Table S.9) in 2003: "People are more likely to use private health care facilities in districts with higher population density," not surprising since there are more private than public health facilities per surface area. Further the number of private clinics grows faster than the number of public clinics with increases in population density. Literacy rates and underreporting percentage per district are statistically correlated: $\rho=0.76, P=0.0001$ in 2003 and $\rho=0.51, P=0.0191$ in 2005 (Table S.9). Hence, it can be said that "literate individuals seem to prefer private health services over public clinics."

\subsection{Spatial Pattern of High-risk Districts}

The total number of reported cases in Bihar is 1.5 times higher in 2005 than in 2003. However, the computed adjusted (for underreporting) incidence rate (Table S.7) in Bihar suggest decreases in the total number of adjusted cases by $28 \%$ from 2003 to 2005.

Adjusted incidence rates changes the ranking of districts (Table S.7). Jahanabad 
experienced the largest decrease while Samastipur the largest increase in adjusted incidence rates from 2003 to 2005. Nine districts and Bihar experienced decreases in adjusted incidences rates from 2003 to 2005. On the other hand, adjusted incidence rates are the highest in Araria (164.4) in 2003 and Madhepura (212.3) in 2005. Bihar's adjusted incidence is 142.9 in 2003 and 98.7 in 2005.

For each district, the case-fraction is computed by dividing the number of cases by total cases (21 districts) per year using both reported and adjusted number of cases. The case-fraction for each district is compared with its population-fraction (population of district divided by populations of 21 districts). We call a district "high-risk" if the case-fraction of the district is greater than its population-fraction. Bihar's high-risk districts are shown separately for 2003 (Figure 2(a)) and 2005 (Figure 2(b)). We marked high-risk districts in the map with stripes if reported data are used (Figure 2). High-risk districts are shaded when adjusted cases data are used (Figure 2). The fractions are reported in percentages in Table S.7. Adjusted incidence data suggest Araria, Begusarai, Madhepura, Madhubani, Saran, and Siwan are high-risk districts common in both years (Figure 2).

\section{- - - - Insert Figure 2 about here. - - - -}

Eight high-risk districts are identified with reported case data and seven (Begusarai, Darbhanga, Madhabani, W. Champaran, Patna, Saran, and Siwan) with adjusted case data in 2003. Reported cases data (2003) classified 4 districts as high-risk districts that adjusted (for underreporting) case data classified as low-risk (Figure 2(a)). Reported incidence data "misidentified" 4 high-risk and 7 low-risk districts in 2003. 
Eleven districts are classified as high-risk using adjusted data but only 9 using reported cases data in 2005. Begusarai, Katihar, Madhubani, Samastipur, and Siwan are high-risk districts under adjusted data but not if reported data are used. Khagaria, E. Champaran, and Vaishali are high-risk districts based on reported data but are removed from this group under adjusted data (Figure 2(b)). The use of reported data "mis-classified" 3 as high-risk and 5 as low-risk districts in 2005.

There are no significant correlations between population size and incidence rates at the district level. The highest correlation is found between adjusted incidence rate and rurality in 2003 (and literacy rate in 2005). In fact, adjusted incidence rates are positively correlated with rurality index $(\rho=0.45, \mathrm{P}=0.04)$ and negatively correlated with literacy rate $(\rho=-0.48, \mathrm{P}=0.01)$.

\subsection{Parameters uncertainty}

The uncertainty in the parameters is modeled through assigned distributions (Figures S.5, and S.6). The distributions of the reproduction numbers are collected in Figure S.5. We observe, for example, that the standard deviation of Bihar's $R_{c}$ values decreased from 4.6 (2003) to 2.0 (2005) but the standard deviation of Bihar's underreporting (percentages) increased from 11.8 in 2003 to 20.3 in 2005 (Figure S.6). The reproductive number values have little effect on underreporting levels if they are significantly larger than one. Underreporting is the most sensitive parameter to changes in incidence rates followed by the variations in life span of the adult sandfly as well as in parameters linked to the transmission of the parasites through sandflies' bites (see Table S.5). 


\section{Discussion}

High rates of frequent inter- and intra- (rural-to-urban) district migration (mostly work-related), lack of access to adequate and accessible public health care facilities, and extreme poverty are some of the factors that facilitate the prevalence of Kalaazar (KA) in Bihar [38]. Large scale underreporting of KA cases in the state is tied in to the lack of reporting requirements for private health facilities. Approximately $73 \%$ of the patients first consult poorly qualified practitioners, most commonly in private practice [45]. A sampling study from a district by Singh et al. (2006) reports that only $12 \%$ of the KA cases in Muzaffarpur, Bihar were reported (and treated) in the public health facilities in 2003 [38]. There have been no studies that systematically quantify Kala-azar underreporting at the district and state level in India. Empirical studies on Kala-azar in Bihar have been carried out with only samples of selected villages in certain districts $[7,38]$. Such statistics, while useful, are unlikely to be representative.

We have quantified KA underreporting in Bihar and its 21 most affected districts using a mathematical model and reported monthly incidence data from 2003 and 2005. Our model takes into account the variability in infectivity, latent period, and treatment duration that occur with regard to Kala-azar in Bihar. This modeling framework, which uses existing reported data to generate adjusted disease trends,

provides the first quantitative measure, as rough as it may be, of the magnitude of the Kala-azar problem in Bihar and its 21 districts.

Model analysis centers around the reproduction number $\left(R_{c}\right)$, defined as the mean number of secondary cases (either human or vector) a typical KA infected case (ei- 
ther human or vector) generates in a susceptible population during the period of infectiousness when KA treatment is available. Analysis shows that the KA exhibits classic threshold behavior, with the infection persisting precisely when $R_{c}>1$. Our estimate of $R_{c}$ for Kala-azar in Bihar using reported data is indeed greater than one (1.3 in 2003 and 2.1 in 2005). These estimates are comparable with the reproduction number estimate (mean of 1.9 for 2000-2004 epidemic of Anthroponotic Cutaneous Leishmaniasis) from Chichaoua province of Morocco [2]. In spite of our $R_{c}$-estimates of some districts being less than one, the disease remains endemic in Bihar state because of the larger values $(>1)$ of $R_{c}$-estimates of most districts. The movement or migration of individuals from endemic districts must have played a role in maintaining disease prevalence in areas where $R_{c}$ is less than one [16].

Model-dependent estimates suggest that the mean case-underreporting for Bihar declined by $17 \%$ from $88 \%$ in 2003 to $73 \%$ in 2005 . Our average estimate of underreporting for the entire state in 2003 is in agreement with that reported by Singh et al. (2006) [38]. Although our mean underreporting seems to be decreasing at the state level, the trend within the state is heterogenous. In some districts average underreporting is as high as $90 \%$ whereas in some others it is as low as $30 \%$. We found that underreporting increased from 2003 to 2005 in only 4 of the 21 districts (Jahanabad, Madhubani, Samastipur, and Katihar).

The model-adjusted (for underreporting) incidence rates are found to be declining in Bihar from 2003 to 2005, even though the reported cases data suggest increases in incidence rates. We found our adjusted incidence rates classify 7 districts as highrisk (Araria, Begusarai, Madhepura, Madhubani, Purnia, Saran, and Siwan) in both 
years 2003 and 2005 .

Reduction in both underreporting and adjusted incidence rates from 2003 to 2005 suggest improvement in Bihar's public health measures. The efforts of the Kala-azar Task Force (formed at the end of 2003) might be behind this decrease. The task force implemented programs under which cost-effective case management tools were made available, referral services were strengthened, monetary relief were provided to patients, and reporting was encouraged [31].

While Kala-azar incidence and case reporting rates in Bihar can clearly be reduced by improving infrastructure in rural areas, providing education for both health care providers and those at risk, reducing population densities and other measures that reduce parasite transmission, these measures all require financial resources. Developing an active case detection surveillance system, which would reduce spread of the infection, would also require resources. The problem of underreporting can also be addressed by making private medical practitioners accountable to the state public health system for proper administration of treatment and reporting; however, while this measure would be considerably less expensive than the others mentioned above, it may be difficult to enforce without community-level support.

More generally, the results of this research highlight the role of mathematical models of vector-borne disease dynamics in quantifying the magnitude of underreporting. The uncertainty in our estimates is high and strongly dependent on the model and naturally on modeling assumptions, but the trends are clear. Underreporting prevents the development and implementation of targeted policies that may be effective in reducing the burden of Kala-azar in Bihar. Furthermore, the frame- 
work identifies areas that would improve if some data were collected and provides a methodology that would allow public health officials to carry out similar studies in India and elsewhere underreporting is an issue.

\section{Acknowledgment}

We would like to thank César V Munayco for help on maps in GIS. Anuj Mubayi thanks Hiroshi Nishura from Utrecht University for insightful comments. This work has been partially supported by a National Science Foundation (Grant DMS-0441114), National Security Agency (Grant H98230-05-1-0097), The Alfred P. Sloan Foundation (through the ASU-Sloan National Pipeline Program in Mathematical Science) and The Office of the Provost of Arizona State University.

\section{References}

[1] Alvar, J., Bashaye, S., Argaw, D., Cruz, I., Aparicio, P., Kassa, A., Orfanos, G., Parreo, F., Babaniyi, O., Gudeta, N., Caavate, C., Bern, C., 2007. Kala-azar outbreak in libo kemkem, ethiopia: Epidemiologic and parasitologic assessment. Am J Trop Med Hyg. 77, 275-82.

[2] Bacaer, N., Guernaoui, S., Sep 2006. The epidemic threshold of vector-borne diseases with seasonality: the case of cutaneous leishmaniasis in chichaoua, morocco. J Math Biol. 53 (3), 421-436.

[3] Badaro, R., Jones, T. C., Carvalho, E. M., Sampaio, D., Reed, S. G., Barral, A., Teixeira, R., Johnson, W. D. J., Dec 1986. New perspectives on a subclinical form of visceral leishmaniasis. J Infect Dis 154 (6), 1003-11. 
[4] Badaro, R., Jones, T. C., Loreno, R., Cerf, B. J., Sampaio, D., Carvalho, E. M., Rocha, H., Teixeira, R., Johnson, W. D. J., Oct 1986. A prospective study of visceral leishmaniasis in an endemic area of brazil. J Infect Dis 154 (4), 639-49.

[5] Bhattacharya, S. K., Sinha, P. K., Sundar, S., Thakur, C. P., Jha, T. K., Pandey, K., Das, V. R., Kumar, N., Lal, C., Verma, N., Singh, V. P., Ranjan, A., Verma, R. B., Anders, G., Sindermann, H., Ganguly, N. K., 2007. Phase 4 trial of miltefosine for the treatment of indian visceral leishmaniasis. The Journal of Infectious Diseases 196, 591-8.

[6] Blower, S. M., Dowlatabadi, H., 1994. Sensitivity and uncertainty analysis of complex models of disease transmission: an hiv model, as an example. Inter Stat Rev. 2, 229-43.

[7] Bora, D., 1999. Epidemiology of visceral leishmaniasis in india. Natl Med J India $12,62-8$.

[8] Burattini, M. N., Coutinho, F. A. B., Lopez, L. F., Massad, E., 1998. Modeling the dynamics of leishmaniasis considering human, animal host and vector populations.. Journal of Biological Systems 6 (4), 337-356.

[9] Cattand, P., Desjeux, P., Guzmn, M. G., J Jannin, A. K., Mdici, A., Musgrove, P., Nathan, M. B., Shaw, A., Schofield, C. J., 2006. Disease Control Priorities in Developing Countries, ed.., 2nd Edition. New York: Oxford University Press., Ch. Tropical Diseases Lacking Adequate Control Measures: Dengue, Leishmaniasis, and African Trypanosomiasis, pp. 451-466. 
[10] Census-Of-India, 2001. Statistics of Bihar. Census of India.

[11] Chakraborty, S., Dutta, M., Srivastava, L., Barui, K. C., Mandal, S. P., 1982. Current outbreaks of kala-azar in malda and murshidabad districts of west bengal - an epidemiological evaluation. Journal of Communicable Diseases 14 (4), $263-73$.

[12] Chaves, L. F., Hernandez, M. J., 2004. Mathematical modelling of american cutaneous leishmaniasis: Incidental hosts and threshold conditions for infection persistence. Acta Trop 92, 245-252.

[13] Chowell, G., Castillo-Chavez, C., Fenimore, P. W., Kribs-Zaleta, C., Arriola, L., Hyman, J. M., 2004. Model parameters and outbreak control for sars. Emerg. Inf. Dis. 10 (7), 1258-63.

[14] Chowell, G., Diaz-Duenas, P., Miller, J. C., Alcazar-Velazco, A., Hyman, J. M., Fenimore, P. W., Castillo-Chavez, C., Aug 2007. Estimation of the reproduction number of dengue fever from spatial epidemic data. Math. Biosci. 208 (2), 571-8.

[15] Courtenay, O., Quinnell, R. J., Garcez, L. M., Shaw, J. J., Dye, C., 2002. Infectiousness in a cohort of brazilian dogs: Why culling fails to control visceral leishmaniasis in areas of high transmission. The Journal of Infectious Diseases $186,1314-1320$.

[16] De-La-Pava, E., 2006. Modelacion mathematica de la participacion del humano como reservorio de la leishmania (viannia) en el pacifico de columbia. Ph.D. thesis, Universidad Del Valle, Columbia. 
[17] Deshingkar, P., Kumar, S., Chobey, H. K., Kumar, D., December 2006. The role of migration and remittances in promoting livelihoods in bihar. Overseas Development Institute, London.

[18] Desjeux, P., 1999. Global control and leishmania hiv co-infection. Clinics in Dermatology 17 (3), 317-25.

[19] Dye, C., 1992. Leishmaniasis epidemiology : theory catches up. Parasitology 104, S7-S18.

[20] Dye, C., 1996. The logic of visceral leishmaniasis control. Am. J. Trop. Med. Hyg. 55 (2), 125-130.

[21] Dye, C., Wolpert, D. M., 1988. Earthquakes, influenza and cycles of indian kalaazar. Transactions of the Royal Society of Tropical Medicine and Hygiene 82 (6), $843-850$.

[22] Esteva, L., Vargas, C., 1998. Analysis of a dengue disease transmission model. Mathematical Biosci. 150, 131-151.

[23] Feng, Z., Xu, D., Zhao, W., 2007. Epidemiological models with nonexponentially distributed disease stages and applications to disease control. Bull. Math. Biol. 69, 1511-1536.

[24] Ferro, C., Crdenas, E., Corredor, D., Morales, A., Munstermann, L. E., Mar/Apr 1998. Life cycle and fecundity analysis of lutzomyia shannoni (dyar) (diptera: Psychodidae). Mem Inst Oswaldo Cruz, Rio de Janeiro, 93 (2), 195-9. 
[25] Garg, R., Dube, A., Mar 2006. Animal models for vaccine studies for visceral leishmaniasis. Indian Journal of Medical Research 123, 439-54.

[26] Guerin, P., Olliaro, P., Sundar, S., Boelaert, M., Croft, S. L., Desjeux, P., Wasunna, M. K., Bryceson, A. D., 2002. Visceral leishmaniasis: Current status of control diagnosis, and treatment, and a proposed research and development agenda. Lancet Infect Dis 2, 494-501.

[27] Herwaldt, B., 1999. Leishmaniasis. Lancet 354, 1191-9.

[28] Koella, J., Boete, C., 2003. A model for the coevolution of immunity and immune evasion in vector-borne diseases with implications for the epidemiology of malaria. AM NAT 161, 698-707.

[29] Marwaha, M., Sarode, S., Gupta, R. K., Garewal, G., Dash, S., 1991. Clinicohematological characteristics in patients with kala azar. Tropical and Geographical Medicine . 43, 357-62.

[30] Molina, R., Gradoni, L., Alvar., J., 2003. Hiv and the transmission of leishmania. Ann. Trop. Med. Parasitol. 97 (Supp. No. 1), S29-S45.

[31] National-Vector-Borne-Disease-Control-Programme, January 2009. http://www.nvbdcp.nic.in.

[32] Palatnik-De-Sousa, C. B., Batista-De-Melo, L. M., Borja-Cabrera, G. P., Palatnik, M., Lavor, C. C., 2004. Improving methods for epidemiological control of canine visceral leishmaniasis based on a mathematical model. impact on the incidence of the canine and human disease. An Acad Bras Cienc 76 (3), 583-593. 
[33] Ranjan, A., Sur, D., Singh, V. P., Siddique, N. A., Manna, B., Lal, C. S., Sinha, P. K., Kishore, K., Bhattacharya, S. K., 2005. Risk factors for indian kala-azar. Am J Trop Med Hyg. 73 (1), 74-78.

[34] Roberts, M. T. M., 2006. Current understandings on the immunology of leishmaniasis and recent developments in prevention and treatment. British Medical Bulletin. 75 and 76, 115-130.

[35] Sacks, D. L., Perkins, P. V., 1984. Identification of an infective stage of leishmania promastigotes. Science 223, 1417-1419.

[36] Sacks, D. L., Perkins, P. V., 1985. Development of infective stage leishmania promastigotes within phlebotomine sand flies. Am J Trop Med Hyg 34, 456459.

[37] Singh, R. K., Pandey, H. P., Sundar, S., 2006. Visceral leishmaniasis (kala-azar): challenges ahead. Indian Journal of Medical Research 123, 331-44.

[38] Singh, S. P., Reddy, D. C. S., Rai, M., Sundar, S., June 2006. Serious underreporting of visceral leishmaniasis through passive case reporting in bihar, india. Tropical Medicine and International Health 11 (6), 899-905.

[39] Srinivasan, R., Panicker, K. N., 1993. Laboratory observations on the biology of the phlebotomid sandfly, phlebotomus papatasi. Southeast Asian J Trop Med Public Health 24 (3), 536-9.

[40] Sud, A., Varma, N., Marwaha, R. K., Patel, F. M., Trehan, A., Singh, S., 
Varma, S., Oct 2004. Visceral leishmaniasis in a non-endemic area of india. Tropical Doctor 34 (4), 247-9.

[41] Sundar, S., Nov 2001. Drug resistance in indian visceral leishmaniasis. Tropical Medicine and International Health 6 (11), 849-54.

[42] Sundar, S., 2003. Indian kala-azar - better tools needed for diagnosis and treatment. Journal of Postgraduate Medicine. 49 (1), 29-30.

[43] Sundar, S., Jha, T. K., Thakur, C. P., Sinha, P. K., Bhattacharya, S. K., 2007. Injectable paromomycin for visceral leishmaniasis in india. NEJM 356, 25712581.

[44] Sundar, S., Mondal, D., Rijal, S., Bhattacharya, S., Ghalib, H., Kroeger, A., Boelaert, M., Desjeux, P., Richter-Airijoki, H., Harms, G., 2008. Implementation research to support the initiative on the elimination of kala azar from bangladesh, india and nepal - the challenges for diagnosis and treatment. Tropical Medicine \& International Health 13 (1), 2-5.

[45] Sundar, S., Thakur, B. B., Tandon, A. K., Agrawal, N. R., Mishra, C. P., Mahapatra, T. M., Singh, V. P., January 1994. Clinicoepidemiological study of drug resistance in indian kala - azar. BMJ., 308-307.

[46] The Times of India, April 27 2008. The times of india. Scientists find kala-azar protein, online newspaper article.

[47] van-den Driessche, P., Watmough, J., 2002. Reproduction numbers and sub- 
threshold endemic equilibria for compartmental models of disease transmission. Mathematical Biosciences 180, 29-48.

[48] Williams, B. G., Dye, C., 1997. Infectious disease persistence when transmission varies seasonally. Math Biosci. 145 (1), 77-88.

[49] Wonham, M., Lewis, M., Renclawowicz, J., van-den Driessche, P., 2006. Conflicting predictions arise from underlying assumptions in host-vector disease models: a case study in west nile virus dynamics. Ecology Letters 9, 706-72.

[50] World-Bank, 2001. India raising the sights. better health systems for indias poor. health nutrition and population sector unit, india, south asia region. Tech. rep., The World Bank.

[51] World-Health-Organization, 1996. Manual of Visceral Leishmaniasis Control.

[52] World-Health-Organization, 1998. Leishmania \& HIV in Gridlock. Geneva: WHO/Joint United Nations Programme on HIV/AIDS.

[53] World-Health-Organization, 2002. World Health Report 2002: Reducing Risks, Promoting Healthy Life.

[54] World-Health-Organization, 2003. World Health Report 2003: Shaping the future.

[55] World-Health-Organization, 2004. The Leishmaniases and Leishmania/HIV coinfections: WHO Fact sheet No. 116. 
[56] World-Health-Organization, 2004. Control of Leishmaniasis. Report of a WHO Expert Committee.

[57] World-Health-Organization, 2004. Report of the Scientific Working Group meeting on Leishmaniasis. 
Table 1: Statistics of demographic and socio-economic variables in 2001 for the 21 districts of the state of Bihar on which our study focuses [10].

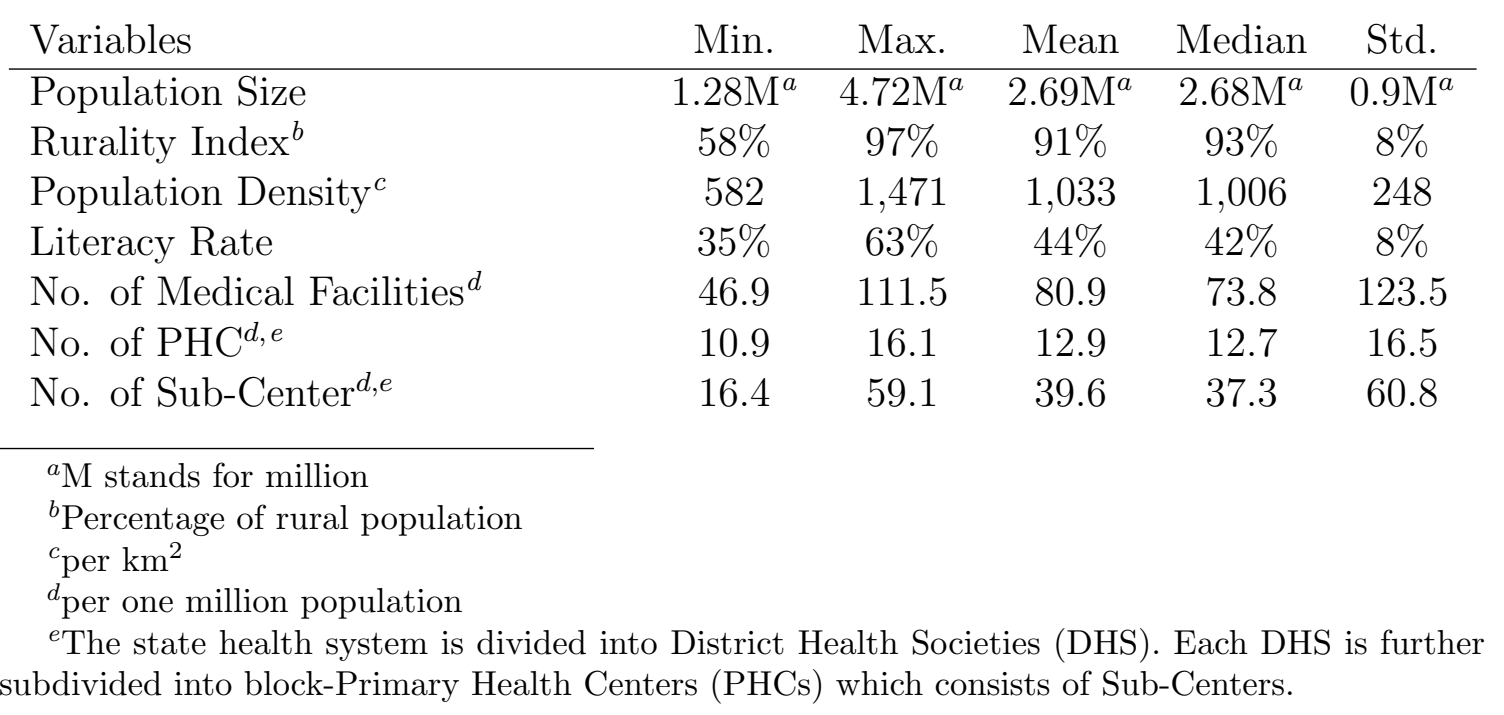


Table 2: Model parameter notation, definition, estimates and their corresponding sources.

\begin{tabular}{|c|c|c|c|c|c|}
\hline Definition & Parameters & $\begin{array}{c}\text { Mean } \\
\text { (months) }\end{array}$ & $\begin{array}{c}\text { Std. } \\
\text { (months) }\end{array}$ & $\begin{array}{l}\text { No. of } \\
\text { stages }\end{array}$ & Ref. \\
\hline Life span of humans & $1 / \mu_{h}$ & 722.4 & - & - & {$[10]$} \\
\hline $\begin{array}{l}\text { Treatment period in gov- } \\
\text { ernment health facilities }\end{array}$ & $1 / \alpha_{h}$ & 0.76 & 0.21 & $k_{1} \approx 13$ & estm. \\
\hline $\begin{array}{l}\text { Treatment period in pri- } \\
\text { vate health facilities }\end{array}$ & $1 / \widetilde{\alpha}_{h}$ & 1.02 & 0.28 & $k_{2} \approx 13$ & estm. \\
\hline Latent period & $1 / \gamma_{h}$ & 4.00 & 1.01 & $n_{1} \approx 16$ & {$[51]$} \\
\hline Infectious period & $1 / \eta_{h}$ & 3.80 & 3.55 & $m_{1} \approx 2$ & {$[40]$} \\
\hline $\begin{array}{l}\text { Proportion of patients } \\
\text { choosing private clinics }\end{array}$ & $1-\mathrm{p}$ & 0.88 & - & - & {$[38]$} \\
\hline Life span of adult sandfly & $1 / \mu_{v}$ & 0.47 & 0.42 & - & {$[39]$} \\
\hline Latent period in Sandfly & $1 / \gamma_{v}$ & 0.17 & 0.03 & $n_{2} \approx 25$ & {$[36]$} \\
\hline
\end{tabular}




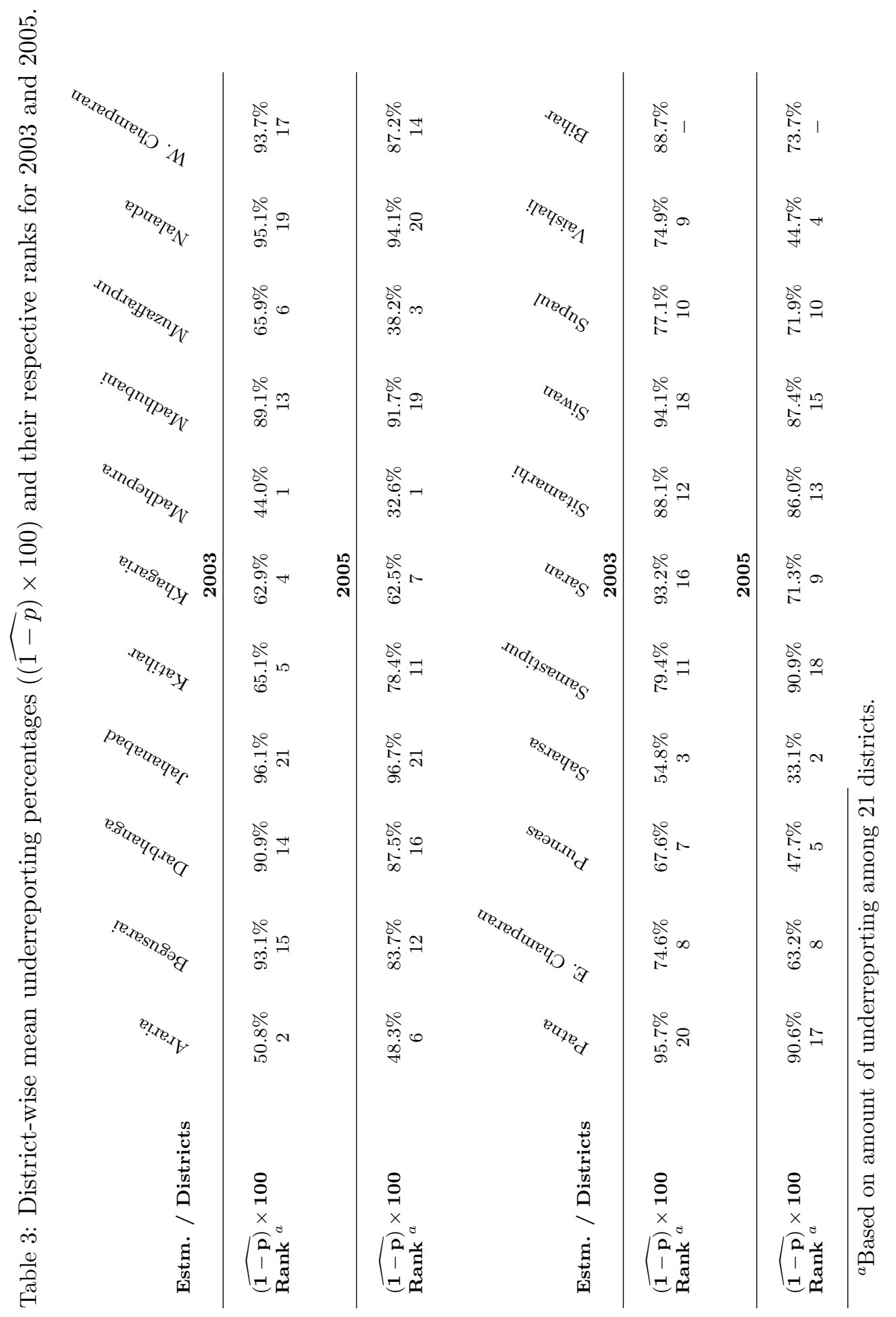




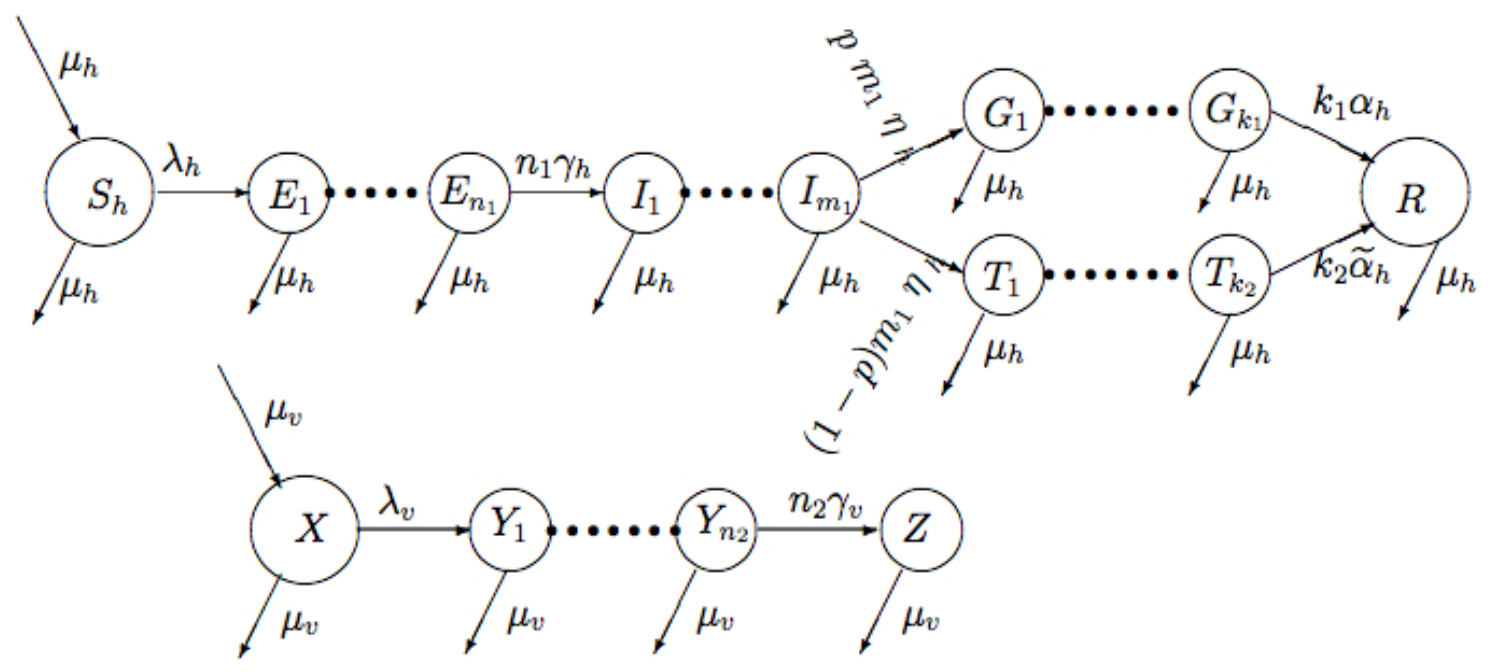

Figure 1: A flow chart of the state progression between epidemiological classes that includes interacting human and vector populations. 


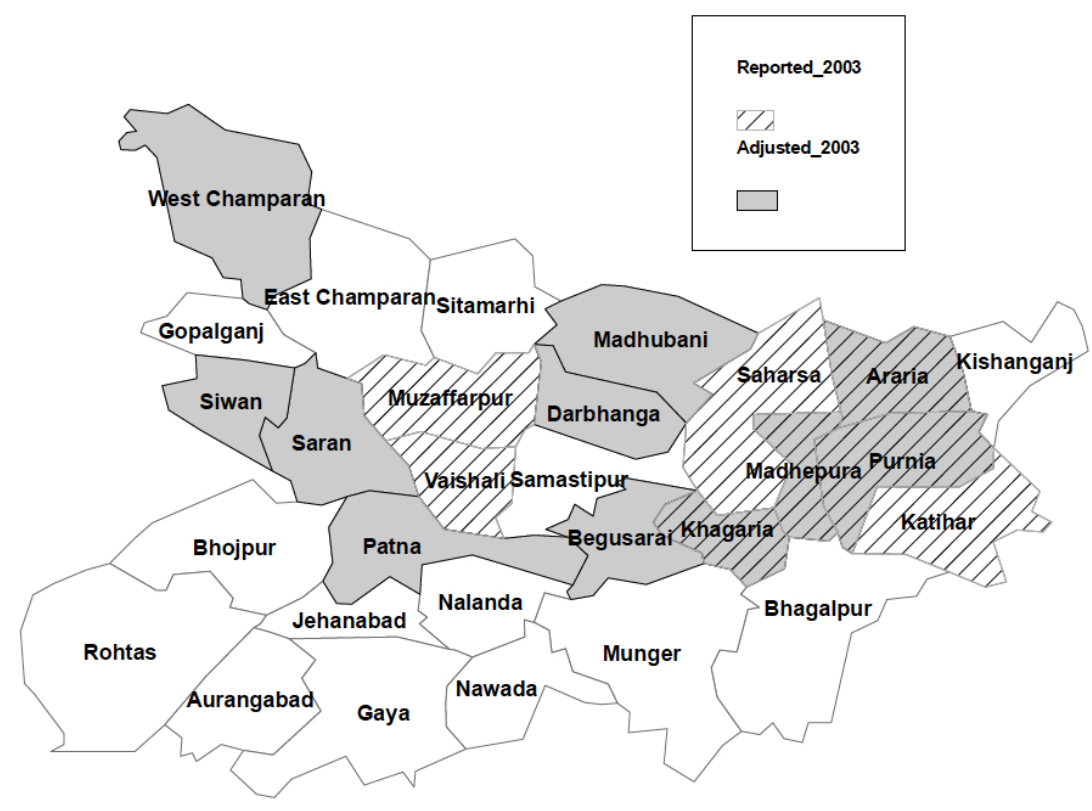

(a) Shaded or stripes districts are Kala-azar high-risk districts in 2003.

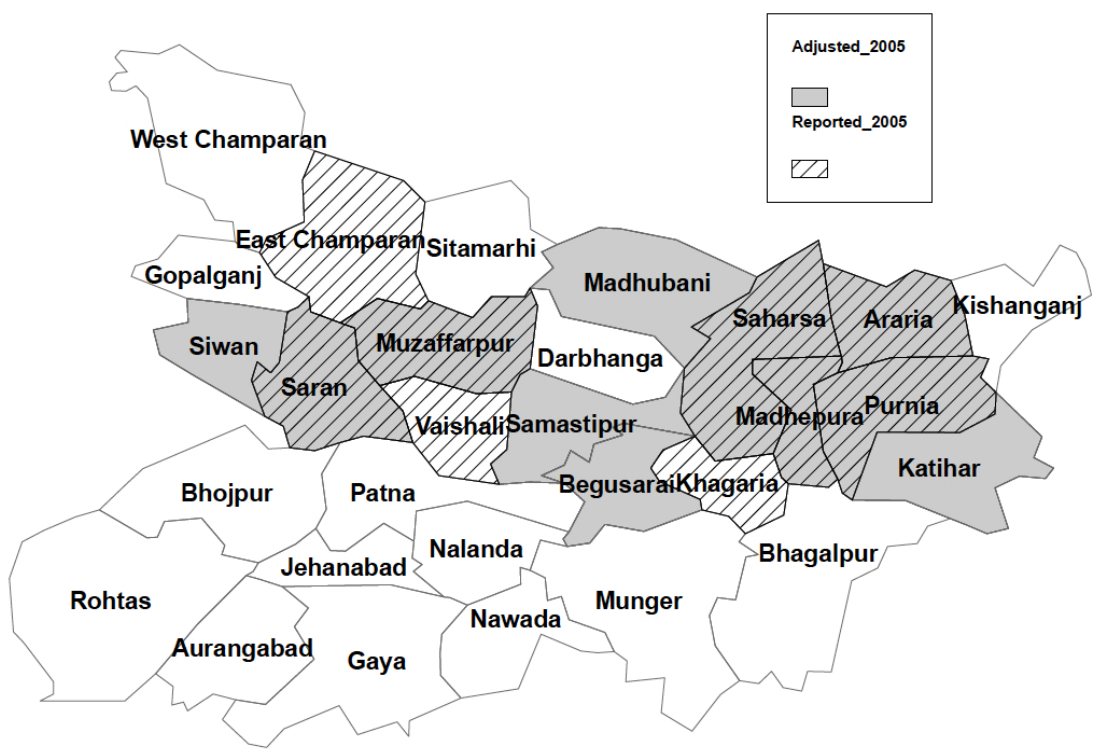

(b) Shaded or stripes districts are Kala-azar high-risk districts in 2005.

Figure 2: Spatial pattern of Kala-azar: drawn using the proportion of population and the proportion of cases (reported and underreporting-adjusted) in the districts. 


\section{Supplementary Document}

\section{Transmission Dynamics and Underreporting of Kala-azar in the Indian State of Bihar: Mubayi A., Castillo-Chavez C., Chowell G., Kribs-Zaleta C., Siddiqui N.A., Kumar N., Das P.}

\section{S.1. Model Description}

For simplicity we assume that all individuals and vectors in our framework are homogeneous and mix uniformly in their respective populations. Individuals are born into the population as susceptible $(S)$ at a constant rate $\mu N$ (where $\mathrm{N}$ is the size of the population) and live for an exponentially distributed time with mean $1 / \mu$ years. In this population we model the spread of Kala-azar (assuming disease deaths are negligible). Once an individual gets infected he/she moves through the latent stages $E_{i}, i=1, \ldots, n_{1}$. He/she is latent for a $\Gamma\left(n_{1}, n_{1} \gamma_{h}\right)$-distributed time after which he/she becomes infectious and remains so for a period modeled by a $\Gamma\left(m_{1}, m_{1} \eta_{h}\right)$-distribution. This means that the expected length of the latent period is $1 / \gamma_{h}$, its standard deviation is $1 /\left(\gamma_{h} \sqrt{n_{1}}\right)$, and the squared coefficient of variation is $1 / n_{1}$. The length of the average infectious period is $1 / \eta_{h}$, with a standard deviation $1 /\left(\eta_{h} \sqrt{m_{1}}\right)$, and the squared coefficient of variation $1 / m_{1}$. If $n_{1}>0$, each infected individual passes through $n_{1}$ stages before becoming infectious, spending an exponentially distributed time in each stage which is independent of time spent in every other stage. Thus, the latent period is the sum of $n_{1}$ independent exponentially distributed parameters each having mean $1 /\left(n_{1} \gamma_{h}\right)$. If $n_{1}=0$, an infected individual immediately becomes infective, the latent period is zero. If $n_{1}=1$ then latent period 
has an exponential distribution with rate parameter $1 / \gamma_{h}$. Once becoming infectious, each individual passes through $m_{1}$ stages $\left(I_{i}, i=1, \ldots, m_{1}\right)$ such that at each stage he/she is equally capable of spreading the infection. The infectious period is thus the sum of $m_{1}$ independent exponential parameters each having mean $1 /\left(m_{1} \eta_{h}\right)$.

The model defined here uses the well-known fact that a $\Gamma(n, v)$-distribution is equivalent to the distribution of the sum of $n$ independent $\operatorname{Exp}(v)$-distribution. A schematic diagram describing the transmission process is given in Figure 1. Using these definitions and notation we arrive at the following non-linear model:

$$
\begin{aligned}
\frac{d S}{d t} & =\mu_{h} N_{h}-F_{h}(t) S_{h}-\mu_{h} S_{h}, \\
\frac{d E_{1}}{d t} & =F_{h}(t) S_{h}-n_{1} \gamma_{h} E_{1}-\mu_{h} E_{1}, \\
\frac{d E_{i_{1}}}{d t} & =n_{1} \gamma_{h} E_{i_{1}-1}-n_{1} \gamma_{h} E_{i_{1}}-\mu_{h} E_{i_{1}}, \quad 2 \leq i_{1} \leq n_{1} \\
\frac{d I_{1}}{d t} & =n_{1} \gamma_{h} E_{n_{1}}-m_{1} \eta_{h} I_{1}-\mu_{h} I_{1}, \\
\frac{d I_{j_{1}}}{d t} & =m_{1} \eta_{h} I_{j_{1}-1}-m_{1} \eta_{h} I_{j_{1}}-\mu_{h} I_{j_{1}}, \quad 2 \leq j_{1} \leq m_{1} \\
\frac{d G_{1}}{d t} & =p m_{1} \eta_{h} I_{m_{1}}-k_{1} \alpha_{h} G_{1}-\mu_{h} G_{1}, \\
\frac{d G_{l_{1}}}{d t} & =k_{1} \alpha_{h} G_{l_{1}-1}-k_{1} \alpha_{h} G_{l_{1}}-\mu_{h} G_{l_{1}}, \quad 2 \leq l_{1} \leq k_{1} \\
\frac{d T_{1}}{d t} & =(1-p) m_{1} \eta_{h} I_{m_{1}}-k_{2} \widetilde{\alpha}_{h} T_{1}-\mu_{h} T_{1}, \\
\frac{d T_{l_{2}}}{d t} & =k_{2} \widetilde{\alpha}_{h} T_{l_{2}-1}-k_{2} \widetilde{\alpha}_{h} T_{l_{2}}-\mu_{h} T_{l_{2}}, \quad 2 \leq l_{2} \leq k_{2} \\
\frac{d R}{d t} & =k_{1} \alpha_{h} G_{k_{1}}+k_{2} \widetilde{\alpha}_{h} T_{k_{2}}-\mu_{h} R_{h},
\end{aligned}
$$




$$
\begin{aligned}
\frac{d X}{d t} & =\mu_{v} N_{v}-F_{v}(t) X-\mu_{v} X \\
\frac{d Y_{1}}{d t} & =F_{v}(t) X-n_{2} \gamma_{v} Y_{1}-\mu_{v} Y_{1}, \\
\frac{d Y_{i_{2}}}{d t} & =n_{2} \gamma_{v} Y_{i_{2}-1}-n_{2} \gamma_{v} Y_{i_{2}}-\mu_{v} Y_{i_{2}}, \quad 2 \leq i_{2} \leq n_{2} \\
\frac{d Z}{d t} & =n_{2} \gamma_{v} Y_{n_{2}}-\mu_{v} Z
\end{aligned}
$$

where $N_{h}=S+\sum_{i_{1}=1}^{n_{1}} E_{i_{1}}(t)+\sum_{j_{1}=1}^{m_{1}} I_{j_{1}}(t)+\sum_{l_{1}=1}^{k_{1}} G_{l_{1}}(t)+\sum_{l_{2}=1}^{k_{2}} T_{l_{2}}(t)+R$ and $N_{v}=X+\sum_{i_{2}=1}^{n_{2}} Y_{i_{2}}(t)+Z$ are constant.

\section{S.2. Existence and Uniqueness of Endemic State}

Setting the right hand side of the System (S.4)-(S.5) to zero we get

$$
\begin{aligned}
0 & =\mu_{h} N_{h}-\lambda_{h} \frac{Z}{N_{v}} S_{h}-\mu_{h} S_{h} \\
0 & =\lambda_{h} \frac{Z}{N_{v}} S_{h}-a_{1} E_{1} \\
R & =\frac{k_{1} \alpha_{h}}{\mu_{h}} G_{k_{1}}+\frac{k_{2} \widetilde{\alpha}_{h}}{\mu_{h}} T_{k_{2}}
\end{aligned}
$$




$$
\begin{aligned}
E_{i_{1}} & =\frac{n_{1} \gamma_{h}}{a_{1}} E_{i_{1}-1} \quad \text { for } 2 \leq i_{1} \leq n_{1} \\
I_{1} & =\frac{n_{1} \gamma_{h}}{a_{2}} E_{n_{1}} \\
I_{j_{1}} & =\frac{m_{1} \eta_{h}}{a_{2}} I_{j_{1}-1} \quad \text { for } 2 \leq j_{1} \leq m_{1} \\
G_{1} & =\frac{p m_{1} \eta_{h}}{b_{1}} I_{m_{1}} \\
G_{l_{1}} & =\frac{k_{1} \alpha_{h}}{b_{1}} G_{l_{1}-1} \\
T_{1} & =\frac{(1-p) m_{1} \eta_{h}}{b_{2}} I_{m_{1}} \\
T_{l_{2}} & =\frac{k_{2} \widetilde{\alpha}_{h}}{b_{2}} T_{l_{2}-1} \quad \text { for } 2 \leq l_{1} \leq k_{1}
\end{aligned}
$$

$$
\begin{aligned}
0 & =\mu_{v} N_{v}-\lambda_{v} \frac{\sum_{j_{1}=1}^{m_{1}} I_{j_{1}}}{N_{h}} X-\mu_{v} X \\
0 & =\lambda_{v} \frac{\sum_{j_{1}=1}^{m_{1}} I_{j_{1}}}{N_{h}} X-c Y_{1} \\
Y_{i_{2}} & =\frac{n_{2} \gamma_{v}}{c} Y_{i_{2}-1} \quad \text { for } 2 \leq i_{2} \leq n_{2} \\
Z & =\frac{n_{2} \gamma_{v}}{\mu_{v}} Y_{n_{2}}
\end{aligned}
$$

where $a_{1}=n_{1} \gamma_{h}+\mu_{h}, a_{2}=m_{1} \eta_{h}+\mu_{h}, b_{1}=k_{1} \alpha_{h}+\mu_{h}, b_{2}=k_{2} \widetilde{\alpha}_{h}+\mu_{h}$ and $c=n_{2} \gamma_{v}+\mu_{v}$. 
Adding Equations (S.6) and (S.7) and also Equations (S.16) and (S.17) we get

$$
\begin{aligned}
E_{1} & =\frac{\mu_{h}}{a_{1}}\left(N_{h}-S_{h}\right) \\
Y_{1} & =\frac{\mu_{v}}{c}\left(N_{v}-X\right)
\end{aligned}
$$

Simplifying Equations (S.9) and (S.18) recursively we get

$$
\begin{aligned}
& E_{i_{1}}=\left(\frac{n_{1} \gamma_{h}}{a_{1}}\right)^{i_{1}-1} E_{1} \quad \text { for } 2 \leq i_{1} \leq n_{1} \\
& Y_{i_{2}}=\left(\frac{n_{2} \gamma_{v}}{c}\right)^{i_{2}-1} Y_{1} \quad \text { for } 2 \leq i_{2} \leq n_{2} \text {. }
\end{aligned}
$$

A similar expression can be obtained from Equations (S.11), (S.13) and (S.15). Hence, using a simplified expression of Equation (S.22) in Equation (S.10) we get

$$
I_{1}=\left(\frac{n_{1} \gamma_{h}}{a_{2}}\right)\left(\frac{n_{1} \gamma_{h}}{a_{1}}\right)^{n_{1}-1} E_{1}
$$

Similarly we can get $G_{1}, T_{1}$, and $Z$ where the first two are a function of $I_{1}$, and $Z$ is a function of $Y_{1}$. In fact $Z$ is a function of $X$ (using Equation (S.21)). Substituting this final expression of $Z$ in Equation (S.6) we get

$$
\frac{S_{h}}{N_{h}}=\frac{\mu_{h}}{\lambda_{h} M_{2}\left(1-\frac{X}{N_{v}}\right)+\mu_{h}}
$$

where $M_{2}=P_{3}^{n_{2}}$ and $P_{3}=\frac{n_{2} \gamma_{v}}{n_{2} \gamma_{v}+\mu_{v}}$. Similarly using Equations (S.16), (S.24) and (S.20) we get

$$
\frac{S_{h}}{N_{h}}=1-\frac{\mu_{v}\left(1-\frac{X}{N_{v}}\right)}{\lambda_{v} M_{3} \frac{X}{N_{v}}}
$$


where $M_{3}=A B, A=\left(P_{2}\right)^{n_{1}} \times\left(1-P_{1}\right), B=\sum_{i=0}^{m_{1}-1}\left(P_{1}\right)^{i}, P_{1}=\frac{m_{1} \eta_{h}}{m_{1} \eta_{h}+\mu_{h}}$ and $P_{2}=\frac{n_{1} \gamma_{h}}{n_{1} \gamma_{h}+\mu_{h}}$.

Equating Equations (S.26) and (S.25) and simplifying we get

$$
u^{2}-\left(1+c_{1}\right) u+c_{1}=0
$$

where $u=\frac{X}{N_{v}}, c_{1}=\frac{1-A_{1}}{B_{1}}, B_{1}=\frac{\lambda_{h} M_{2}}{\lambda_{h} M_{2}+\mu_{h}}$, and $A_{1}=\frac{\lambda_{v} M_{3}}{\lambda_{v} M_{3}+\mu_{v}}$. This quadratic equation has two roots 1 and $c_{1}$. Clearly, $A_{1}, B_{1}, P_{1}, P_{2}, P_{3}, P_{6}$ and $P_{6}$ are positive and less than one. This implies that $c_{1}$ is positive. Hence, both roots of the quadratic equation are positive. Since $u=X / N_{v}$ lies between 0 and 1 , the root $c_{1}>1$ will not have biological relevance.

If $c_{1}=1$ then both roots of the quadratic equation are the same and we obtain the Disease-Free State (DFS). The DFS of the model is

$$
\begin{array}{r}
\left(s^{f}, e_{1}^{f}, \ldots, e_{n_{1}}^{f}, i_{1}^{f}, \ldots, i_{m_{1}}^{f}, g_{1}^{f}, \ldots, g_{k_{1}}^{f}, t_{1}^{f}, \ldots, t_{k_{2}}^{f}, r^{f} ; x^{f}, y_{1}^{f}, \ldots, y_{n_{2}}^{f}, z^{f}\right)= \\
(1,0, \ldots, 0,0 \ldots 0,0 \ldots 0,0 \ldots 0,0 ; 1,0 \ldots 0,0)
\end{array}
$$

If $c_{1}<1$ (or $A_{1}+B_{1}>1$ or $R_{0}>1$; Equation (1)) then the system has two steady states: DFS and Endemic State (ES). This is because the endemic state components corresponding to susceptible humans, $s^{e}$, and susceptible vectors, $x^{e}$, are less than 
one. The ES is given by

$$
\begin{aligned}
s^{e} & =\frac{1-B_{1}}{A_{1}}, & & \\
e_{1}^{e} & =\left(1-P_{2}\right)\left(1-s^{e}\right), & & \\
e_{i_{1}}^{e} & =\left(P_{2}\right)^{i_{1}-1}\left(1-P_{2}\right)\left(1-s^{e}\right), & & \\
i_{1}^{e} & =A\left(1-s^{e}\right), & & \\
i_{j_{1}}^{e} & =\left(P_{1}\right)^{j_{1}-1} A\left(1-s^{e}\right), & & \\
g_{1}^{e} & =\left(\frac{p m_{1} \eta_{h}}{k_{1} \alpha_{h}+\mu_{h}}\right)\left(P_{1}\right)^{m_{1}-1} A\left(1-s^{e}\right), & & \\
g_{l_{1}}^{e} & =\left(\frac{p m_{1} \eta_{h}}{k_{1} \alpha_{h}+\mu_{h}}\right)\left(P_{6}\right)^{l_{1}-1}\left(P_{1}\right)^{m_{1}-1} A\left(1-s^{e}\right), & & \text { for } 2 \leq l_{1} \leq k_{1} \\
t_{1}^{e} & =\left(\frac{(1-p) m_{1} \eta_{h}}{k_{2} \widetilde{\alpha}_{h}+\mu_{h}}\right)\left(P_{1}\right)^{m_{1}-1} A\left(1-s^{e}\right), & & \\
t_{l_{2}}^{e} & =\left(\frac{(1-p) m_{1} \eta_{h}}{k_{2} \widetilde{\alpha}_{h}+\mu_{h}}\right)\left(P_{7}\right)^{l_{2}-1}\left(P_{1}\right)^{m_{1}-1} A\left(1-s^{e}\right), & & \text { for } 2 \leq l_{2} \leq k_{2} \\
x^{e} & =\frac{1-A_{1}}{B_{1}}, & & \\
y_{1}^{e} & =\left(1-P_{3}\right)\left(1-x^{e}\right), & & \\
y_{i_{2}}^{e} & =\left(P_{3}\right)^{i_{2}-1}\left(1-P_{3}\right)\left(1-x^{e}\right) & & \\
z^{e} & =\left(P_{3}\right)^{n_{2}}\left(1-x^{e}\right) . & &
\end{aligned}
$$

$$
\begin{aligned}
& \text { where } A=\left(P_{2}\right)^{n_{1}} \times\left(1-P_{1}\right), B=\sum_{i=0}^{m_{1}-1}\left(P_{1}\right)^{i}, A_{1}=\frac{\lambda_{v} A B}{\lambda_{v} A B+\mu_{v}}, B_{1}=\frac{\lambda_{h} P_{3}^{n_{2}}}{\lambda_{h} P_{3}^{n_{2}+\mu_{h}}}, P_{1}= \\
& \left(\frac{m_{1} \eta_{h}}{m_{1} \eta_{h}+\mu_{h}}\right), P_{2}=\left(\frac{n_{1} \gamma_{h}}{n_{1} \gamma_{h}+\mu_{h}}\right), P_{3}=\left(\frac{n_{2} \gamma_{v}}{n_{2} \gamma_{v}+\mu_{v}}\right), P_{6}=\left(\frac{k_{1} \alpha_{h}}{k_{1} \alpha_{h}+\mu_{h}}\right) \text { and } P_{7}=\left(\frac{k_{2} \widetilde{\alpha}_{h}}{k_{2} \widetilde{\alpha}_{h}+\mu_{h}}\right) .
\end{aligned}
$$




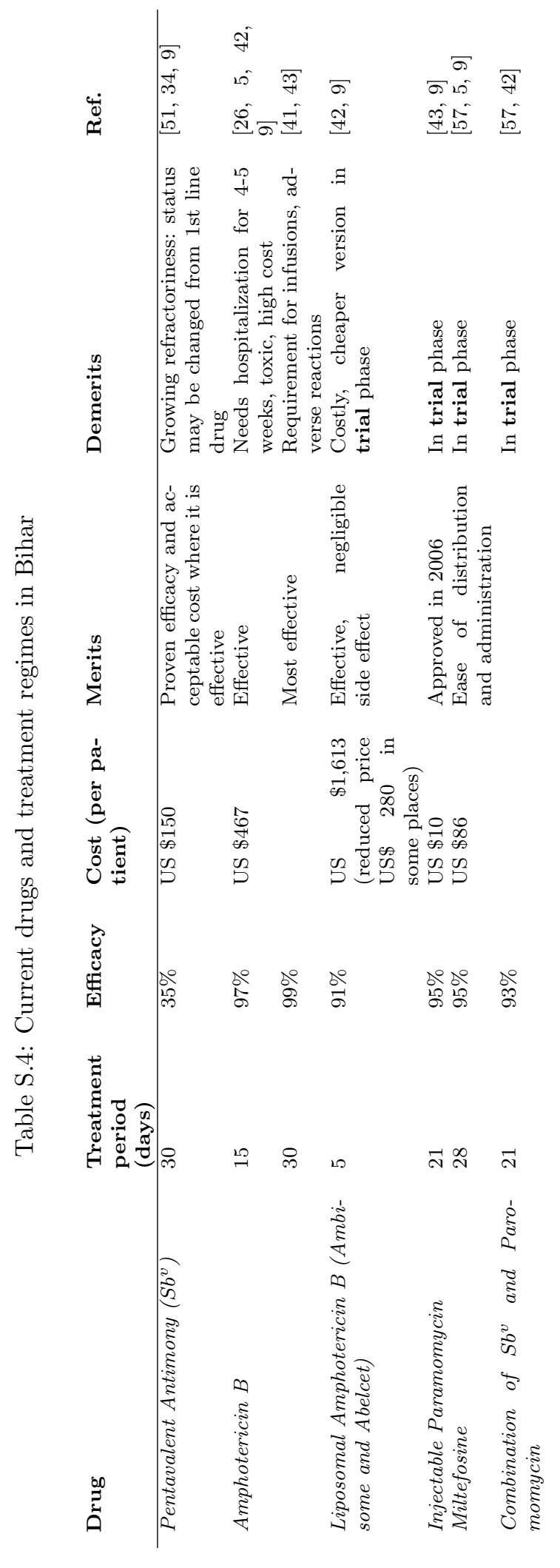




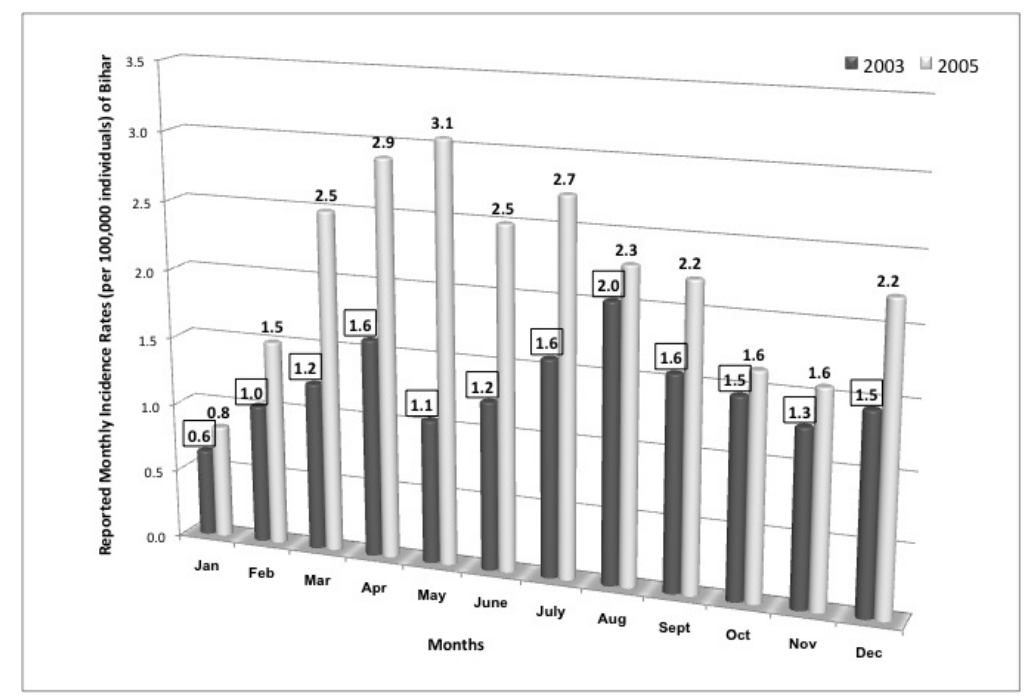

Figure S.3: Monthly Reported Incidence Rates (per 100,000 individuals) for 2003 and 2005 in Bihar, India.

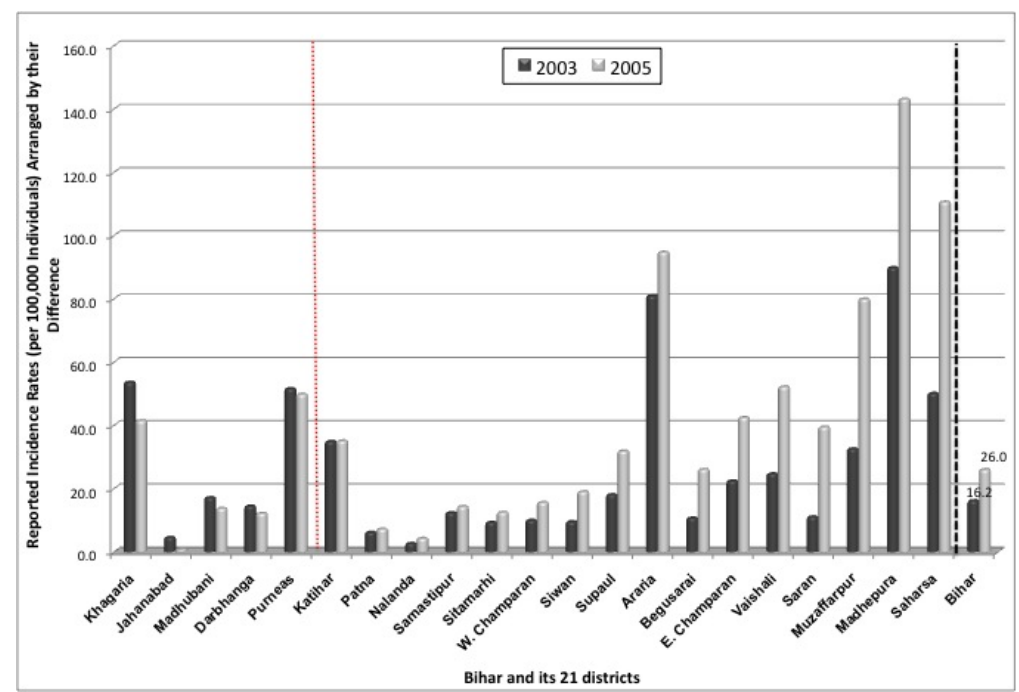

Figure S.4: District-wise Reported Incidence Rate (per 100,000 individuals) for 2003 and 2005 in Bihar, India. 
Table S.5: Pearson correlation coefficients between underreporting and some model parameters. Significant figures are bolded in the table.

\begin{tabular}{c|ccccccc} 
& $\mu_{v}$ & $\lambda_{h}$ & $\lambda_{v}$ & $m_{1}$ & $n_{1}$ & $n_{2}$ & Reported_cases \\
& \multicolumn{7}{c}{$\mathbf{2 0 0 3}$} \\
\hline$r$ & $\mathbf{0 . 1 6}$ & $\mathbf{0 . 1 3}$ & $\mathbf{0 . 1 1}$ & $\mathbf{- 0 . 0 9}$ & 0.01 & -0.02 & $\mathbf{- 0 . 2 2}$ \\
$\mathrm{P}$ & $<0.05$ & $<0.05$ & $<0.05$ & $<0.05$ & 0.29 & 0.12 & $<0.05$ \\
& \multicolumn{7}{c}{} \\
& \multicolumn{7}{c}{$\mathbf{2 0 0 5}$} \\
\hline$r$ & $\mathbf{- 0 . 1 4}$ & $\mathbf{0 . 1 9}$ & $\mathbf{0 . 1 5}$ & $\mathbf{- 0 . 0 7}$ & 0.02 & -0.05 & $\mathbf{- 0 . 2 1}$ \\
$\mathrm{P}$ & $<0.05$ & $<0.05$ & $<0.05$ & $<0.05$ & 0.51 & 0.29 & $<0.05$
\end{tabular}

Table S.6: Parameter estimates for Bihar State. $R_{c}^{*}$ is computed by taking average over reproduction number estimates of 21 districts whereas $R_{c}$ estimates by using total state (Bihar) reported cases data. Sign + indicate best fit estimates (by using Chi-square goodness-of-fit statistical test). Column 1 represents number of data points used in the curve fitting.

\begin{tabular}{ccccccc} 
Parameter & $\lambda_{h}$ & $\lambda_{h}$ & $E_{h_{1}}(0)$ & $I_{h_{1}}(0)$ & $R_{c}^{*}$ & $R_{c}$ \\
\hline No. of data pts. & \multicolumn{6}{c}{$\mathbf{2 0 0 3}$} \\
\hline 6 & 0.77 & 1.69 & 1017 & 2502 & 2.04 & 1.48 \\
8 & 0.66 & 0.96 & 1153 & 2513 & 1.43 & 1.84 \\
$\mathbf{9}^{+}$ & 0.94 & 1.13 & 1266 & 2498 & 1.33 & 1.80 \\
10 & $\mathbf{0 . 7 1}$ & $\mathbf{0 . 9 8}$ & $\mathbf{1 4 2 2}$ & $\mathbf{2 4 9 1}$ & $\mathbf{1 . 1 1}$ & $\mathbf{1 . 2 7}$ \\
11 & 0.83 & 0.92 & 1499 & 2499 & 0.99 & 1.02 \\
12 & 0.49 & 0.93 & 1587 & 2495 & 0.92 & 0.91 \\
No. of data pts. & 0.59 & 0.94 & 1617 & 2493 & 0.91 & 0.90 \\
\hline 6 & 0.31 & 0.20 & 3257 & 4976 & 0.98 & 8.25 \\
7 & 0.24 & 0.73 & 2922 & 5020 & 0.84 & 4.73 \\
8 & 0.74 & 1.17 & 2696 & 5058 & 0.72 & 3.10 \\
9 & 0.25 & 1.40 & 2677 & 5062 & 0.60 & 2.53 \\
$\mathbf{1 0}$ & $\mathbf{0 . 2 6}$ & $\mathbf{0 . 4 6}$ & $\mathbf{2 6 8 8}$ & $\mathbf{5 0 6 6}$ & $\mathbf{0 . 5 1}$ & $\mathbf{2 . 0 8}$ \\
11 & 0.25 & 0.48 & 2672 & 5067 & 0.46 & 1.79 \\
12 & 0.33 & 0.81 & 2357 & 5096 & 0.58 & 1.61
\end{tabular}


Frequency Distribution of Parameters for Bihar State Using 2003 Data
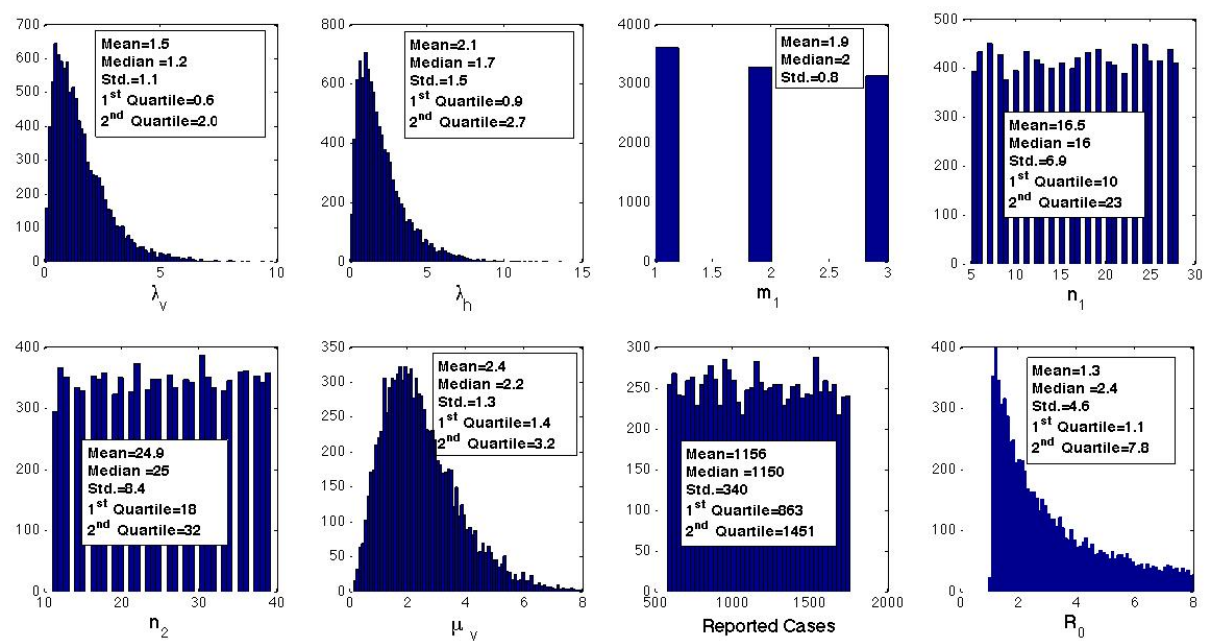

(a) Distributions of parameters obtained from 2003 incidence data.

Frequency Distribution of Parameters for Bihar State Using 2005 Data
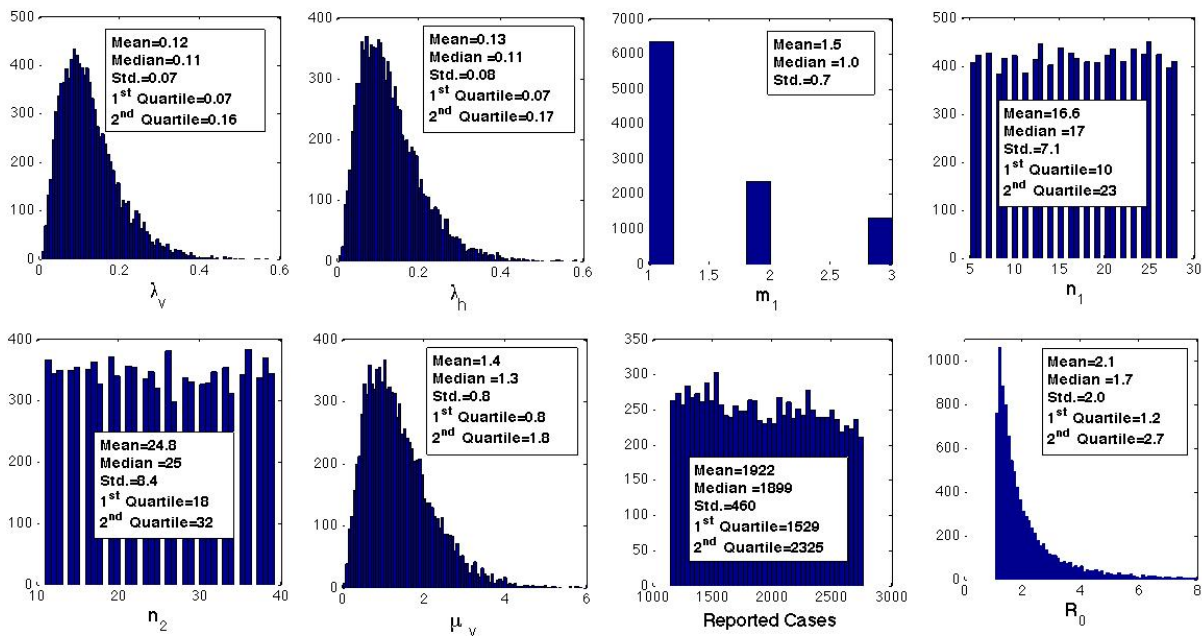

(b) Distributions of parameters obtained from 2005 incidence data.

Figure S.5: Distributions of parameters including $R_{c}$ for the whole state of Bihar. 


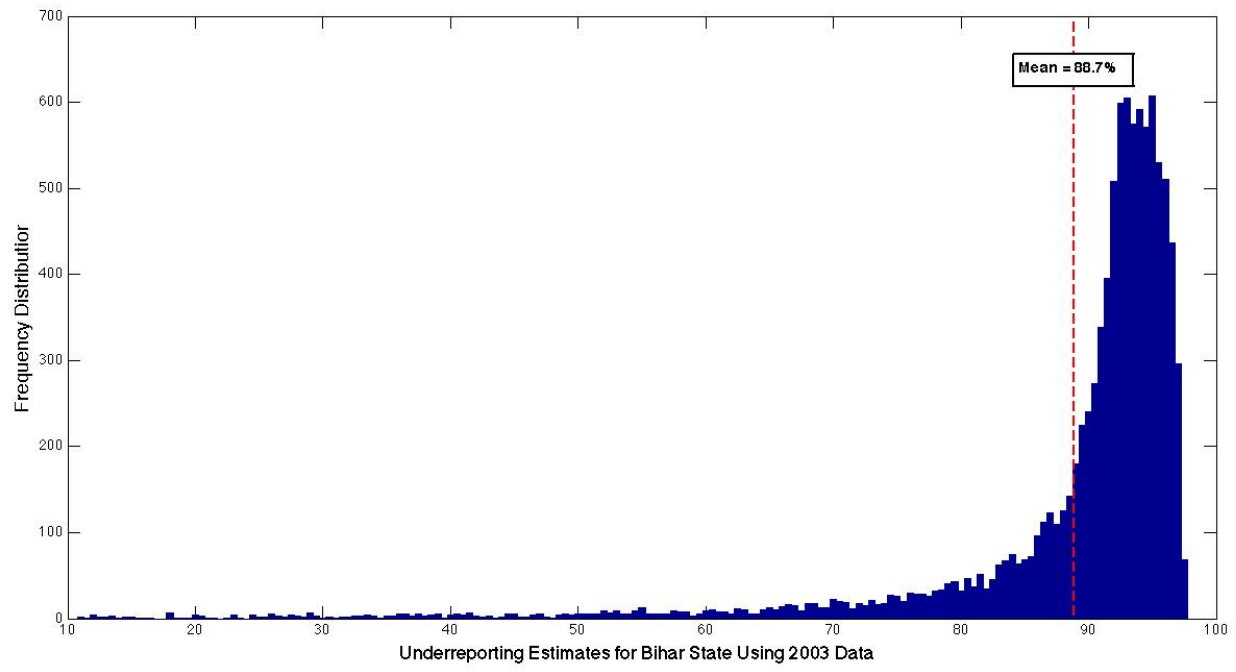

(a) Distribution with $\hat{p}=88.7$ and standard deviation 11.8 obtained using 2003 incidence data.

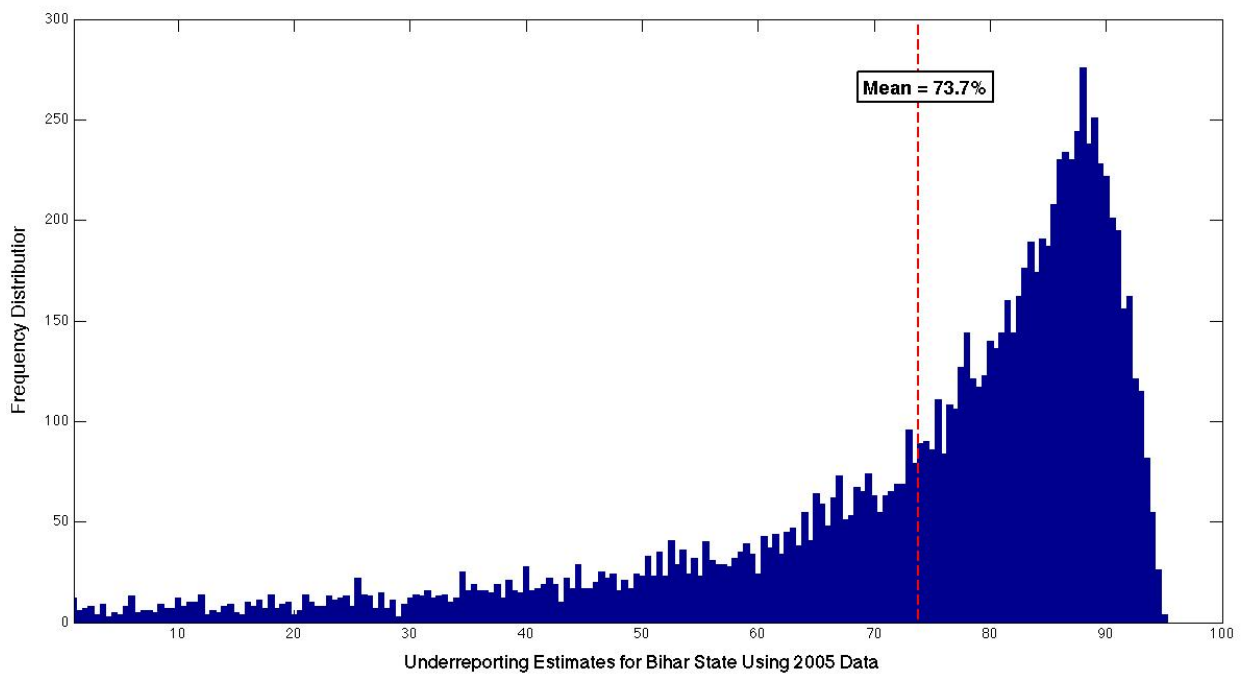

(b) Distribution with $\hat{p}=73.7$ and standard deviation 20.3 obtained using 2005 incidence data.

Figure S.6: Distributions of underreporting parameter, $p$, for the whole state of Bihar. 


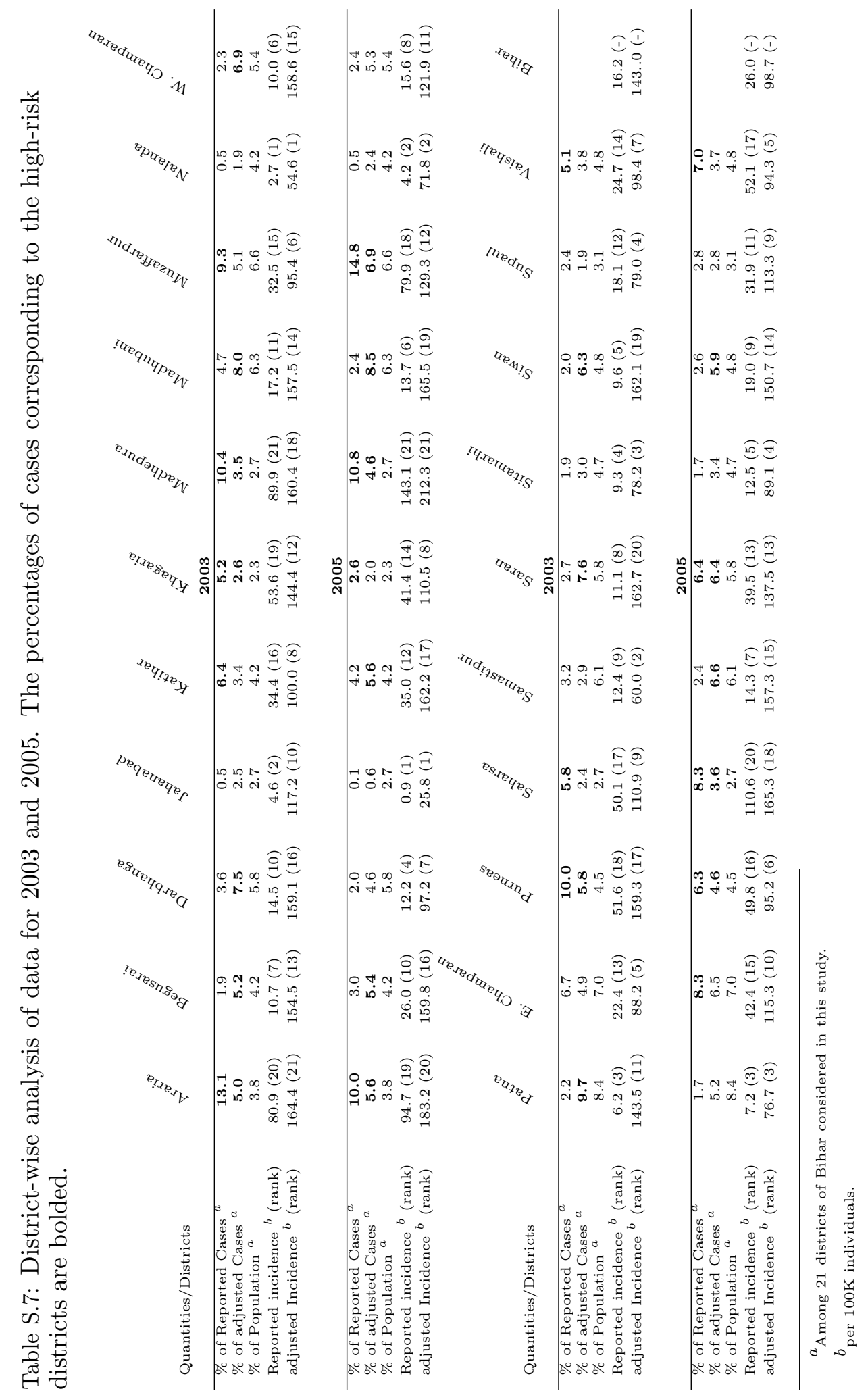




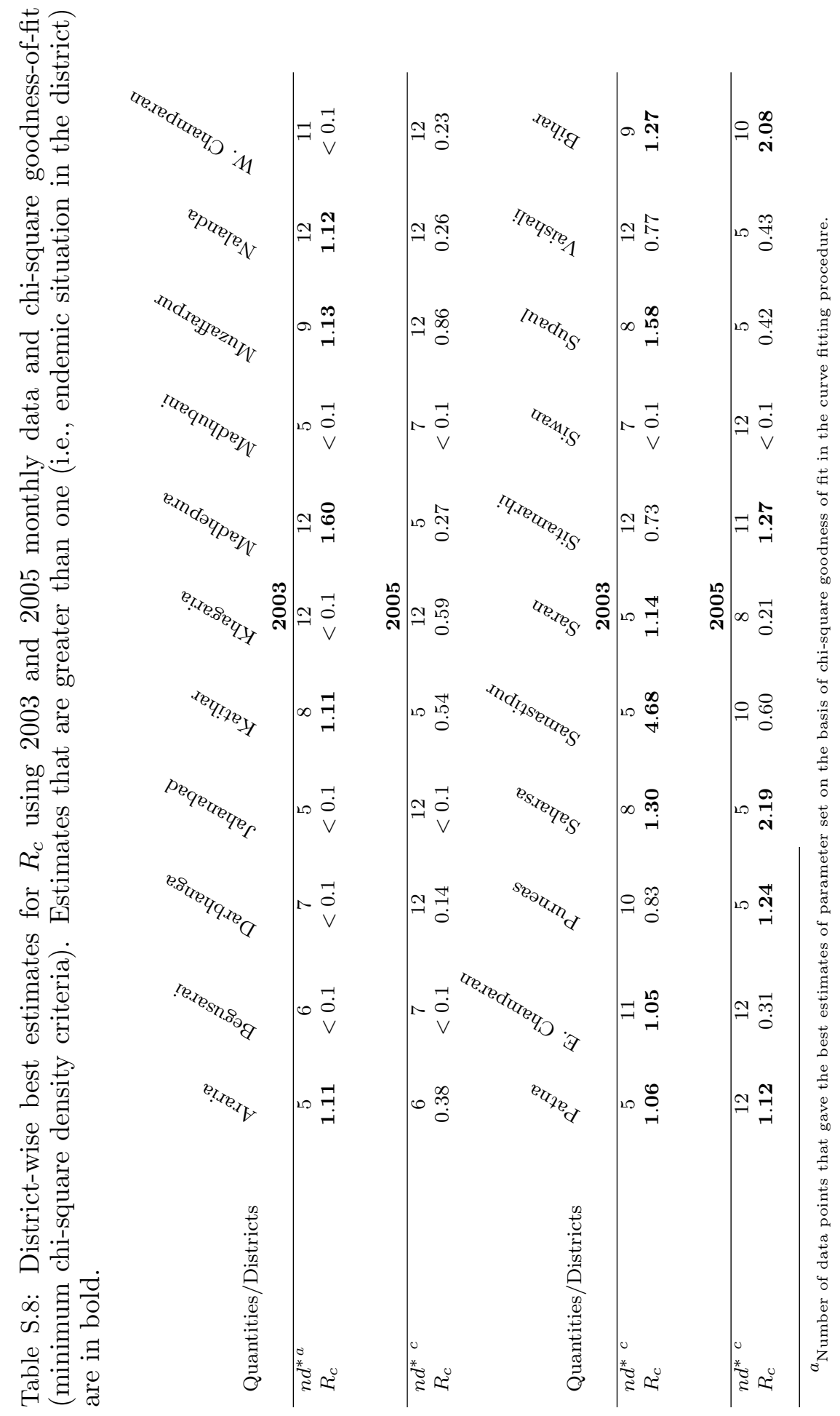


Table S.9: Spearman correlation coefficients between underreporting and demographic and socioeconomic variables. Significant figures are hi-lighted in bold.

\begin{tabular}{c|ccccccc} 
& Pop. $^{a}$ & Pop. Denisty & Rural Pop. & $\begin{array}{c}\text { Literacy Rate } \\
\mathbf{2 0 0 3}\end{array}$ & No. of PHC & No. of S-C & No. of M. F. $^{d}$ \\
\hline$\rho$ & 0.33 & $\mathbf{0 . 4 7}$ & 0.27 & $\mathbf{0 . 7 6}$ & 0.27 & 0.27 & 0.18 \\
$\mathrm{P}$ & 0.15 & 0.03 & 0.24 & 0.00 & 0.24 & 0.24 & 0.42 \\
& & & & $\mathbf{2 0 0 5}$ & & 0.15 & 0.24 \\
\hline$\rho$ & 0.26 & 0.25 & 0.23 & $\mathbf{0 . 5 1}$ & 0.51 & 0.29 & 0.85
\end{tabular}

${ }^{a}$ Size of population.

${ }^{b}$ Number of Primary Health Centres (PHC) per 1 million population.

${ }^{c}$ Number of Sub-Centers per 1 million population.

${ }^{d}$ Number of Medical Facilities per 1 million population. 